\title{
Search for charged Higgs bosons decaying into a top and a bottom quark in the all-jet final state of pp collisions at $\sqrt{s}=13 \mathrm{TeV}$
}

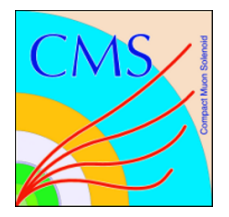

\section{The CMS collaboration}

E-mail: cms-publication-committee-chair@cern.ch

ABSTRACT: A search for charged Higgs bosons $\left(\mathrm{H}^{ \pm}\right)$decaying into a top and a bottom quark in the all-jet final state is presented. The analysis uses LHC proton-proton collision data recorded with the CMS detector in 2016 at $\sqrt{s}=13 \mathrm{TeV}$, corresponding to an integrated luminosity of $35.9 \mathrm{fb}^{-1}$. No significant excess is observed above the expected background. Model-independent upper limits at 95\% confidence level are set on the product of the $\mathrm{H}^{ \pm}$production cross section and branching fraction in two scenarios. For production in association with a top quark, limits of 21.3 to $0.007 \mathrm{pb}$ are obtained for $\mathrm{H}^{ \pm}$masses in the range of 0.2 to $3 \mathrm{TeV}$. Combining this with a search in leptonic final states results in improved limits of 9.25 to $0.005 \mathrm{pb}$. The complementary $s$-channel production of an $\mathrm{H}^{ \pm}$is investigated in the mass range of 0.8 to $3 \mathrm{TeV}$ and the corresponding upper limits are 4.5 to $0.023 \mathrm{pb}$. These results are interpreted using different minimal supersymmetric extensions of the standard model.

KEYwords: Beyond Standard Model, Hadron-Hadron scattering (experiments), Higgs physics

ArXiv EPrint: 2001.07763 


\section{Contents}

1 Introduction 1

2 The CMS detector 3

3 Event samples and simulation $\quad 3$

4 Object reconstruction $\quad 4$

5 Search strategy $\quad \mathbf{5}$

$\begin{array}{ll}5.1 & \text { Event categories in the boosted analysis }\end{array}$

$\begin{array}{lll}5.2 & \text { Event selection in the resolved analysis } & 7\end{array}$

6 Backgrounds $\quad 9$

6.1 Background estimations in events with boosted $\mathrm{W}$ boson and top quark candidates 9

$\begin{array}{lll}\text { 6.2 Background estimations in events with resolved top quarks } & 10\end{array}$

$\begin{array}{lll}7 & \text { Systematic uncertainties } & 11\end{array}$

8 Results and interpretation $\quad 12$

9 Combination with the leptonic final states $\quad 16$

$\begin{array}{ll}10 \text { Summary } & 17\end{array}$

$\begin{array}{lr}\text { The CMS collaboration } & 28\end{array}$

\section{Introduction}

The observation of a Higgs boson [1-5] has motivated new areas of study at the CERN LHC, including precision measurements of its interactions with standard model (SM) particles [68], searches for decays to new particles, and studies of the Higgs boson self interactions. Often, models beyond the SM contain an extended Higgs sector. Minimal extensions known as two Higgs doublet models (2HDMs) [9-12] include a second complex Higgs doublet that leads to five physical particles: two charged Higgs bosons, $\mathrm{H}^{ \pm}$, two neutral scalars, $\mathrm{h}$ and $\mathrm{H}$, and one neutral pseudoscalar, $\mathrm{A}$. The $2 \mathrm{HDM}$ are further classified according to the couplings of the doublets to fermions. One of the popular 2HDMs is the minimal supersymmetric standard model (MSSM) $[13,14]$ where one doublet couples to up quarks and the other to down quarks and charged leptons (Type-II 2HDM). In these models, the lightest CP-even Higgs boson can align with the properties of the SM, while the additional 

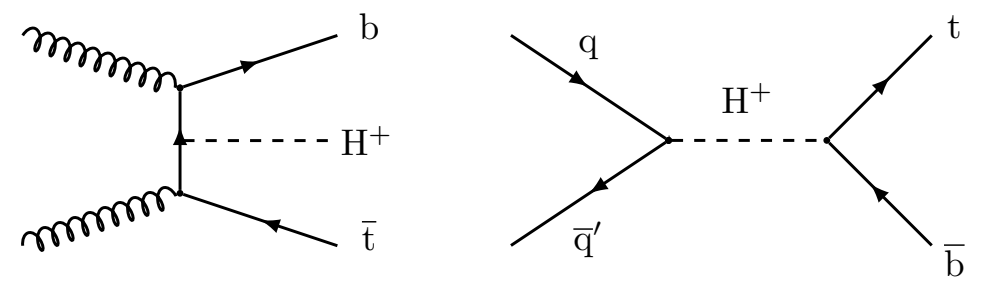

Figure 1. The LO diagrams for production of a heavy charged Higgs boson, showing the production with a top and a bottom quark (4FS) (left) and via an $s$-channel process (right).

Higgs bosons can appear at or below the $\mathrm{TeV}$ scale [15]. At tree level, the production and decay of the $\mathrm{H}^{ \pm}$depends on its mass $\left(m_{\mathrm{H}^{ \pm}}\right)$and the ratio of the vacuum expectation values of the neutral components of the two Higgs doublets $(\tan \beta)$. No fundamental charged-scalar boson is present in the SM, and the discovery of such a particle would uniquely point to physics beyond the SM.

We report a search for charged Higgs bosons with mass larger than that of the top quark (heavy $\mathrm{H}^{ \pm}$) decaying to a top and bottom quark-antiquark pair $\left(\mathrm{H}^{+} \rightarrow \mathrm{t} \overline{\mathrm{b}}\right)$. The production of the boson in association with a top quark can be described using either a four- (4FS) or a five-flavor (5FS) scheme [16, 17], which yield consistent results. It can also be produced directly via an $s$-channel process. The corresponding leading order (LO) Feynman diagrams are shown in figure 1. Charge-conjugate processes are implied throughout this paper.

Several searches for the signature $\mathrm{H}^{+} \rightarrow t \bar{b}$ by the ATLAS and CMS Collaborations in proton-proton (pp) collisions at center-of-mass energies of 8 and $13 \mathrm{TeV}$ [18-21] have been interpreted in the context of $2 \mathrm{HDMs}$. Results of searches for a light $\mathrm{H}^{ \pm}$produced in the decay $\mathrm{t} \rightarrow \mathrm{H}^{+} \mathrm{b}$ that subsequently decays to $c \overline{\mathrm{s}}$ or $\mathrm{c} \overline{\mathrm{b}}$ are presented in refs. [22, 23]. Limits on the production of an $\mathrm{H}^{ \pm}$using the $\tau^{+} v_{\tau}$ decay channel have also been obtained at center-of-mass energies of 8 and $13 \mathrm{TeV}[18,24-26]$. Charged-current processes from low-energy precision flavor observables, such as tauonic B meson decays and the $\mathrm{b} \rightarrow \mathrm{s} \gamma$ transition, can be affected by the presence of the charged Higgs boson. These results currently provide the best indirect lower limit on $m_{\mathrm{H}^{ \pm}}$in the Type-II $2 \mathrm{HDM}[27,28]$. Complementary searches for additional neutral heavy Higgs bosons decaying to a pair of third generation fermions have been performed by ATLAS and CMS at $\sqrt{s}=8$ and $13 \mathrm{TeV}$ in $\mathrm{t} \overline{\mathrm{t}}, \mathrm{b} \overline{\mathrm{b}}$, and $\tau^{+} \tau^{-}$decay channels $[18,24,29-35]$. The production of $\mathrm{H}^{ \pm}$via vector boson fusion with subsequent decays to $\mathrm{W}$ and $\mathrm{Z}$ bosons is expected in models containing Higgs triplets [36]. These searches are discussed in refs. [37-39].

The results presented here are based on pp collision data collected in 2016 at $\sqrt{s}=$ $13 \mathrm{TeV}$ by the CMS experiment, corresponding to an integrated luminosity of $35.9 \mathrm{fb}^{-1}$. The search investigates all-jet events, targeting a signal containing $\mathrm{W} \rightarrow \mathrm{q} \overline{\mathrm{q}}^{\prime}$ decays, in the decay chains of both the charged Higgs boson and the associated top quark. The all-jet final state provides the largest accessible branching fractions, $\approx 45 \%$ and $\approx 67 \%$ for the top quark associated and the $s$-channel processes. In addition, all the final state objects are detected, enabling full reconstruction of the invariant mass of the $\mathrm{H}^{ \pm}$candidate. This 
analysis is the first to report results in the all-jet $t \bar{b} \rightarrow \mathrm{Wb} \bar{b} \rightarrow$ jjb $\bar{b}$ channel for top quark associated production and $s$-channel production of a charged Higgs boson.

The search targets two distinct event topologies, boosted (for top quark associated and $s$-channel processes) and resolved (top quark associated only). The boosted analysis targets $\mathrm{H}^{ \pm}$bosons with mass $m_{\mathrm{H}^{ \pm}} \gtrsim 5 m_{\text {top }}$. Decay products of $\mathrm{H}^{ \pm}$resonances with mass of $\mathcal{O}(\mathrm{TeV})$ have average transverse momenta $\left(p_{\mathrm{T}}\right)$ of several hundred $\mathrm{GeV}$. As a consequence, the objects emerging from subsequent decays of top quarks are highly collimated jets that may not be fully resolved using the standard clustering algorithm, but can be reconstructed as a single large-radius jet. We therefore use these collimated top quark- or W boson-jet candidates to distinguish signal events. The resolved analysis focuses on less boosted final states where each top quark candidate can be reconstructed from jets associated with $\mathrm{W} \rightarrow \mathrm{jj}$ and one jet identified as originating from the fragmentation of a b quark ("btagged"). Therefore a minimum of seven jets is expected for the associated production channel. The search is sensitive to any narrow resonant charged state that decays to $t \bar{b}$.

Model-independent upper limits on the product of the $\mathrm{H}^{ \pm}$production cross section and branching fraction into a top and a bottom quark $(\sigma \mathcal{B})$ as a function of $m_{\mathrm{H}^{ \pm}}$are presented below. These limits can also be recast into model-dependent limits and interpreted in scenario-specific limits, where the underlying free parameters (e.g., $m_{\mathrm{H}^{ \pm}}$, branching fractions, and $\tan \beta$ ) are fixed by the specific scenario. Beyond the $2 \mathrm{HDM}$ interpretations, this decay mode is relevant in the more general context of exotic resonance searches, motivated by models of $\mathrm{W}^{\prime}$ boson production $[40,41]$.

\section{The CMS detector}

The central feature of the CMS apparatus is a superconducting solenoid of $6 \mathrm{~m}$ internal diameter, providing a magnetic field of $3.8 \mathrm{~T}$. Within the solenoid volume are a silicon pixel and strip tracker, a lead tungstate crystal electromagnetic calorimeter, and a brass and scintillator hadron calorimeter, each composed of a barrel and two endcap sections. Forward calorimeters extend the pseudorapidity $(\eta)$ coverage beyond these barrel and endcap detectors. Muons are detected in gas-ionization chambers embedded in the steel flux-return yoke outside the solenoid. Events of interest are selected using a two-tiered trigger system [42]. The first level, composed of specialized hardware processors, uses information from the calorimeters and muon detectors, while the high-level trigger consists of a farm of processors running a version of the full event reconstruction software optimized for fast processing. A more detailed description of the CMS detector, together with a definition of the coordinate system used and the relevant kinematic variables, can be found in ref. [43].

\section{Event samples and simulation}

The main SM backgrounds in this analysis are multijet events produced exclusively via quantum chromodynamics (QCD) interactions and top quark-antiquark pair production. Other sources of background include single top quark production and $t \overline{\mathrm{t}}+\mathrm{X}$ processes with 
$\mathrm{X}=(\mathrm{W}, \mathrm{Z}, \gamma, \mathrm{H}$, or $\mathrm{t} \overline{\mathrm{t}})$, and also $\mathrm{V}+$ jets $(\mathrm{V}=\mathrm{Z}$ or $\mathrm{W})$, diboson (WZ, ZZ, WW, VH) and triboson processes. The latter group is denoted as the "Electroweak" background below.

Simulated samples are produced using various Monte Carlo (MC) event generators. Signal samples are generated using the 4FS at next-to-leading order (NLO) precision in perturbative QCD with the MADGRAPH5_aMC@NLO v2.3.3 [44] generator for a range of $m_{\mathrm{H}^{ \pm}}$hypotheses from 0.2 to $3 \mathrm{TeV}$. The total cross section for the $\mathrm{H}^{ \pm}$production associated with a top quark is obtained using the Santander matching scheme [17]. Typical values are of the order of $1 \mathrm{pb}$ for $m_{\mathrm{H}^{ \pm}}=0.2 \mathrm{TeV}$, down to about $10^{-4} \mathrm{pb}$ for a mass of $3 \mathrm{TeV}\left[16,45^{-}\right.$ 49]. The $s$-channel signal processes are simulated using LO CoMPHEP 4.5.2 [50] following a $\mathrm{W}_{\mathrm{R}}^{\prime}$ model in the narrow-width approximation, in the mass range from 0.8 to $3 \mathrm{TeV}$ [41]. Branching fractions $\mathcal{B}\left(\mathrm{H}^{+} \rightarrow \mathrm{t} \overline{\mathrm{b}}\right)$ are computed using HDECAY v6.25 [51] for different values of $\tan \beta$.

Both the QCD multijet and V+jets background samples are simulated at LO using

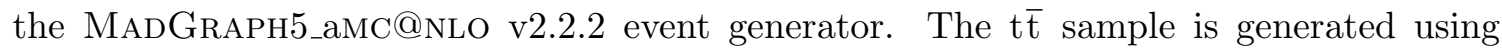
POWHEG v2 [52-54] at NLO in QCD [55], assuming a top quark mass of $172.5 \mathrm{GeV}$. Single top quark events are generated at NLO precision in the $4 \mathrm{FS}$ for the $t$-channel process [56] using POWHEG v2 interfaced with MADSPIN [57] for simulating the top quark decay. The $s$-channel process is simulated using MADGRAPH5_aMC@NLO v2.2.2, while the production of single top quark events via the tW channel is simulated at NLO in the 5FS using POWHEG v1 [58]. The "diagram removal" approach [59] is used to avoid the partial double counting of $\mathrm{tW}$ production vs. $\mathrm{t} \overline{\mathrm{t}}$ production at NLO. The production of $\mathrm{t} \overline{\mathrm{t}}$ in association with W, Z, or $\gamma$ is simulated at NLO using MADGraph5_amC@NLO v2.2.2. The produc-

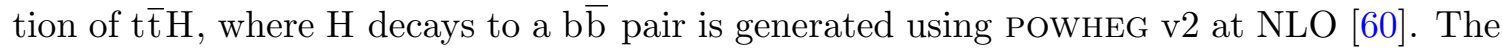
samples are normalized to the most precise available cross section calculations, corresponding most often to next-to-next-to-leading order (NNLO) in QCD and NLO in electroweak corrections [61-73].

Parton distribution functions (PDFs) are modeled using the NNPDF3.0 [74] parametrization. Parton showering and fragmentation are performed using the PYTHIA v8.212 [75] generator. The CUETP8M2T4 [76] tune is used to characterize the underlying event in the $t \overline{\mathrm{t}}$ background, while the CUETP8M1 $[77,78]$ tune is used for all other processes.

The response of the CMS detector for all generated samples is simulated using GEAnT4 v9.4 [79]. Additional pp interactions in the same or nearby bunch crossings (pileup) are generated with PYTHIA v8.212 and superimposed on the hard collisions. In the data collected in 2016, an average of 23 pp interactions occurred per LHC bunch crossing. Simulated events are corrected to produce the pileup distribution in data based on the measured luminosity profile and average measured total inelastic pp cross section [80].

\section{Object reconstruction}

Events are processed using the particle-flow (PF) [81] algorithm, which aims to reconstruct and identify all particles (PF candidates) using the optimal combination of information from the tracker, calorimeters, and muon systems of the CMS detector. 
Electron candidates are identified by matching clusters of energy deposits in the electromagnetic calorimeter to reconstructed charged-particle trajectories in the tracker. A number of selection criteria based on the shower shape, track-cluster matching, and consistency between the cluster energy and track momentum are then applied for the identification of electrons [82]. Muons are reconstructed by requiring consistent hit patterns in the tracker and muon systems [83]. The relative isolation variable for an electron or muon candidate is defined as the scalar sum of the transverse momenta of all PF candidates in a cone around the candidates' trajectory divided by the lepton $p_{\mathrm{T}}$. The cone size depends on the lepton $p_{\mathrm{T}}$, and is bounded between a distance parameter of 0.05 and of 0.2 . Hadronically decaying $\tau$ leptons of $p_{\mathrm{T}} \geq 20 \mathrm{GeV}$, within $|\eta|=2.3$ are reconstructed using the hadron-plus-strip algorithm [84]. The corresponding isolation variable is computed using a multivariate approach, combining information on its identification, isolation and lifetime $[84,85]$.

The primary jet collection is formed by clustering PF candidates using the anti- $k_{\mathrm{T}}$ algorithm $[86,87]$ with a distance parameter of 0.4 . Jet energies are corrected for contributions coming from event pileup [88]. Additional corrections to the jet energy scale [89] are applied to compensate for nonuniform detector response. Jets are required to have $p_{\mathrm{T}}>40 \mathrm{GeV}$ and be contained within the tracker volume of $|\eta|<2.4$. For the resolved analysis, the $p_{\mathrm{T}}$ requirement is relaxed to $30 \mathrm{GeV}$ for subleading jets ranking seventh or lower in $p_{\mathrm{T}}$. In the boosted analysis an additional large-radius jet collection is defined using a distance parameter of 0.8 .

Jets consistent with originating from the decay of a b quark are identified using the combined secondary vertex (CSV) b tagging algorithm [90], at the medium or loose working points. These are defined such that the efficiency to select light-flavor quarks ( $\mathrm{u}, \mathrm{d}$, or s) or gluons as b jets is about $1 \%$ (medium) or $10 \%$ (loose), and the corresponding efficiency for tagging jets from a b (c) quark decay is about 65 or $80 \%$ (10 or $25 \%$ ), respectively. For brevity we refer to jets satisfying the $\mathrm{b}$ tagging criteria as $\mathrm{b}$ jets below.

The scalar $p_{\mathrm{T}}$ sum of all selected jets in an event is denoted as $H_{\mathrm{T}}$, while the missing transverse momentum vector $\vec{p}_{\mathrm{T}}^{\text {miss }}$ is defined as the projection onto the plane perpendicular to the beam axis of the negative vector sum of the momenta of all reconstructed PF candidates [91]. Its magnitude is referred to as $p_{\mathrm{T}}^{\text {miss }}$. Quality requirements are applied to remove a small fraction of events in which detector effects, such as the electronic noise, can affect the $p_{\mathrm{T}}^{\text {miss }}$ reconstruction [91]. The energy scale and resolution corrections applied to jets are propagated to the calculation of $H_{\mathrm{T}}$ and $p_{\mathrm{T}}^{\text {miss }}$.

\section{$5 \quad$ Search strategy}

The analysis aims to reconstruct the full event in order to search for a local enhancement in the top and bottom quark-antiquark invariant mass spectrum.

Because of the large cross section for the QCD multijet background, restrictive trigger requirements are needed to reduce the data recording rate. The data used for this search are collected with an inclusive online selection of $H_{\mathrm{T}}^{\text {trig }}>900 \mathrm{GeV}$, with $H_{\mathrm{T}}^{\text {trig }}$ being defined as the scalar $p_{\mathrm{T}}$ sum of small-radius jets with $p_{\mathrm{T}}^{\text {trig }}>30 \mathrm{GeV}$. Events are also acquired 
with a dedicated large-radius jet trigger requiring $p_{\mathrm{T}}^{\text {trig }}>360 \mathrm{GeV}$ and a mass after jet trimming [92] of at least $30 \mathrm{GeV}$. Furthermore, events satisfying trigger requirements of $H_{\mathrm{T}}^{\text {trig }}>450(400) \mathrm{GeV}$ and six jets with $p_{\mathrm{T}}^{\text {trig }}>40(30) \mathrm{GeV}$ are selected if at least one (two) of them satisfies $\mathrm{b}$ tagging criteria. In the low $m_{\mathrm{H}^{ \pm}}$regions the sensitivity of the all-jet final state is limited by the relatively high trigger thresholds.

Two analyses are performed, each targeting different regions of the signal parameter space. The boosted analysis targets charged Higgs bosons with high mass and utilizes collimated hadronically decaying $\mathrm{W}$ boson or top quark candidates to distinguish signal events. A collection of large-radius jets is used to reconstruct and identify the objects from the decays of boosted $\mathrm{W}$ boson and top quark. In order to discriminate against QCD multijet backgrounds, we exploit both the reconstructed jet mass, which is required to be close to the $\mathrm{W}$ boson or top quark mass, and the two- or three-prong jet substructure (subjets) corresponding to the $\mathrm{W} \rightarrow \mathrm{q} \overline{\mathrm{q}}^{\prime}$ or $\mathrm{t} \rightarrow \mathrm{q} \overline{\mathrm{q}}^{\prime} \mathrm{b}$ decay [93]. The soft-drop algorithm [94] is used to remove soft and wide-angle radiation. The use of soft-drop grooming reduces the resulting jet mass $m_{\mathrm{SD}}$ for QCD multijet events where large jet masses arise from soft-gluon radiation. Finally, because top-quark jets contain a b quark and $\mathrm{W}$ jets do not, additional discrimination power is achieved by applying the CSV algorithm described above to the constituent subjets. The events are categorized according to the number of b-tagged jets to separate sources of SM background and capture signals with both high and low number of $b$ quarks.

The resolved analysis is optimized for charged Higgs bosons with lower masses that decay to moderately boosted top quarks, often identified as three separate small-radius jets, one of which is $\mathrm{b}$ tagged and the other two jets resulting from the $\mathrm{W}$ boson decay. The resolved top quark candidates $\left(\mathrm{t}^{\mathrm{res}}\right)$ are identified using a multivariate boosted decision tree with gradient boost (BDTG) classifier. The classifier exploits properties of the top quark and its decay products such as masses, angular separations, and other kinematic distributions. Additional input variables are quark vs. gluon [95], charm vs. light quark [96], and $b$ tagging discriminator values for each of the three jets. The signal enriched region is defined by requiring the presence of seven or more jets, comprising two resolved top quark candidates and an additional b-tagged jet used to reconstruct the $\mathrm{H}^{ \pm}$candidate.

Both analyses veto the presence of an isolated charged lepton (e or $\mu)$ with $p_{\mathrm{T}} \geq$ $10 \mathrm{GeV}$, or an isolated hadronically decaying tau lepton with $p_{\mathrm{T}} \geq 20 \mathrm{GeV}$. The lepton veto ensures that leptonic final states of $\mathrm{W}$ bosons produced in top quark decays are not considered. These are covered by dedicated analyses [21]. To further reduce the background from semileptonic t $\overline{\mathrm{t}}$ decays, the boosted analysis requires events to have $p_{\mathrm{T}}^{\text {miss }}<200 \mathrm{GeV}$. These requirements also reduce background from any sources containing $\mathrm{W}$ and $\mathrm{Z}$ boson decays.

\subsection{Event categories in the boosted analysis}

Events with at least one b-tagged jet and one identified top quark candidate are considered in the boosted analysis. The $\mathrm{H}^{ \pm}$candidate four-momentum vector is reconstructed as the sum of the four-vectors of the loose-tagged $\mathrm{b}$ jet with highest $p_{\mathrm{T}}$ and the top quark candidate with mass most closely matching $m_{\text {top }}$. A top quark candidate is identified as a 
$\mathrm{t}$ jet or the combination of a $\mathrm{W}$ jet and $\mathrm{a} \mathrm{b}$ jet, excluding the $\mathrm{b}$ jet with highest $p_{\mathrm{T}}$. The $\mathrm{W}(\mathrm{t})$ jet candidates are required to have $65<m_{\mathrm{SD}}<105 \mathrm{GeV}\left(135<m_{\mathrm{SD}}<220 \mathrm{GeV}\right)$, $p_{\mathrm{T}}>200(400) \mathrm{GeV}$, and $|\eta|<2.4$. The hard substructures are identified using the $N$ subjettiness [97] ratios: $\tau_{2} / \tau_{1}<0.6$ for the $\mathrm{W}$ jet and $\tau_{3} / \tau_{2}<0.67$ for the $\mathrm{t}$ jet. We introduce four mutually exclusive categories. The labels "t1b" and "t0b" refer to events containing a large-radius jet identified as a t jet, where at least one, or exactly zero, of the subjets satisfies the medium working point of the b tagging algorithm. In the "wbb" category the top quark candidate is formed from a $\mathrm{W}$ jet and an additional medium-tagged b jet. The "wbj" category relaxes the $b$ tagging requirement on one of the additional jets to satisfy the loose b tagging working point.

The signal is characterized as a peak in the invariant mass distribution $m_{\mathrm{tb}}$ of $\mathrm{H}^{ \pm}$ candidates. This distribution is dominated by background contributions from QCD multijet processes. The expected shape of the $\mathrm{H}^{ \pm}$candidate mass distribution is dominated by the detector resolution and pairing errors, where jets are not correctly matched to the decay products of the boson; the latter is primarily responsible for the sideband on the left of the peak of the invariant mass distribution. The full width at half maximum of the reconstructed MC mass distribution for correct jet assignments is used to describe the mass resolution, and events falling outside this window are used to constrain the background. The mass resolution is consistent among the different event categories. For a charged Higgs boson of mass $1 \mathrm{TeV}$ the resolution is approximately $140 \mathrm{GeV}$. The distribution of the invariant mass $m_{\mathrm{tb}}$ for the $\mathrm{t} 1 \mathrm{~b}$ category is shown in figure 2 for the data, the expected background and for a signal of mass $m_{\mathrm{H}^{ \pm}}=1 \mathrm{TeV}$. To enhance the expected signal to background ratio, data are selected within a window around different charged Higgs boson candidate masses in each of the categories listed above. We then search for an excess of events in the $H_{\mathrm{T}}$ data distribution.

To better separate signal from background, the event categories are further subdivided to exploit differences in jet and b jet multiplicities. For signal events produced in association with a top quark, we expect at least three b quarks in the final state and a large number of extra jets not participating in the reconstruction of the $\mathrm{H}^{ \pm}$. Signal produced in the $s$ channel contains two b quarks and fewer extra jets. We therefore consider different requirements on the number of b-tagged jets: exactly one, exactly two, and at least three. We also distinguish two categories based on the number of additional small-radius jets, less than three $\left(N_{\text {jets }}<3\right)$ or at least three $\left(N_{\text {jets }} \geq 3\right)$ such jets.

The signal-rich regions are analyzed together with signal-depleted regions using a binned maximum likelihood fit to the $H_{\mathrm{T}}$ data distributions that simultaneously determines the contributions from signal and the major background sources.

\subsection{Event selection in the resolved analysis}

A multivariate analysis is employed to select top quark candidates in events containing seven or more jets. We employ a BDTG classifier that is trained on simulated top quarkantiquark pair events using the TMVA package [98]. The signal objects are considered to be three small-radius jet combinations, in which each individual jet is matched to the decay product of a top quark at the generator level. Similarly, background objects are 


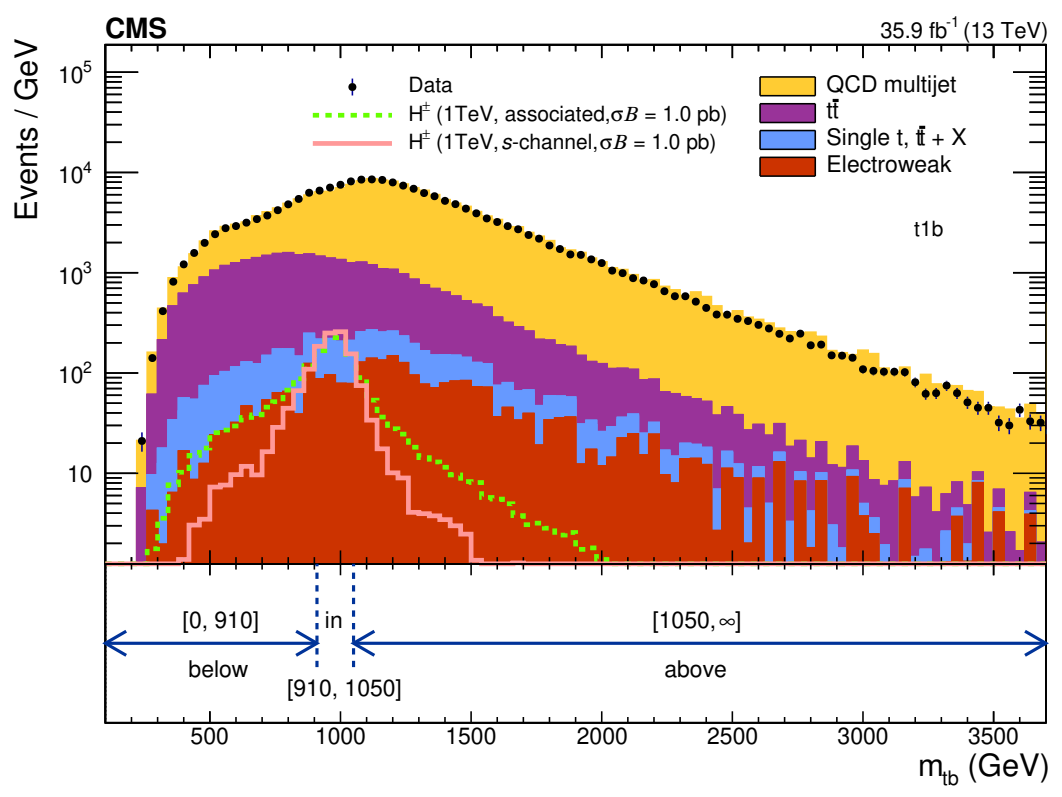

Figure 2. Data and SM background for the event sample with one t jet as a function of the charged Higgs boson candidate mass. The category t1b is shown and the background normalization is fixed to the SM expectation. The signal mass distributions for associated and $s$-channel production of an $\mathrm{H}^{ \pm}$with $m_{\mathrm{H}^{ \pm}}=1 \mathrm{TeV}$ normalized with a cross section times branching fraction of $1 \mathrm{pb}$ are superimposed as open histograms. The signal mass window "in" for associated production is shown together with the sidebands "below" and "above" for the mass hypothesis of $1 \mathrm{TeV}$.

defined as three-jet combinations in which at least one jet is not matched to a top quark decay product. The input variables used for the BDTG training (19 in total), calculated from these jet combinations, are described in detail in ref. [99]. In the BDTG response distribution, values close to -1 are mainly populated by fake top quark candidates from QCD multijet processes, while values close to +1 are dominated by top quark candidates from $t \bar{t}$ or signal events. In this analysis we require resolved top quark candidates to have a BDTG score > 0.4, yielding a signal object efficiency of $92 \%$, and a background object efficiency of $6 \%$.

Events with at least three b-tagged jets passing the CSV medium working point and at least four additional jets are selected. The first top quark candidate is identified by pairing each b-tagged jet with all two-jet combinations and retaining the combination with the highest BDTG value. The same procedure is applied for the second candidate, using only the remaining jets as inputs. To reduce the combinatorial background, we require all the combined three-jet systems to have an invariant mass less than $400 \mathrm{GeV}$.

The efficiency of the BDTG requirement as a function of the $p_{\mathrm{T}}$ of the generated top quark in t $\bar{t}$ events is shown in figure 3 (left), along with the misidentification rate observed in a QCD multijet sample. At the plateau the tagging efficiency reaches $50 \%$. The observed decrease in efficiency in the high- $p_{\mathrm{T}}$ region is due to top quark decay products becoming increasingly collimated, resulting in a jet-to-parton matching inefficiency. The misidentification rate is less than $8 \%$ for the entire $p_{\mathrm{T}}$ range considered. 

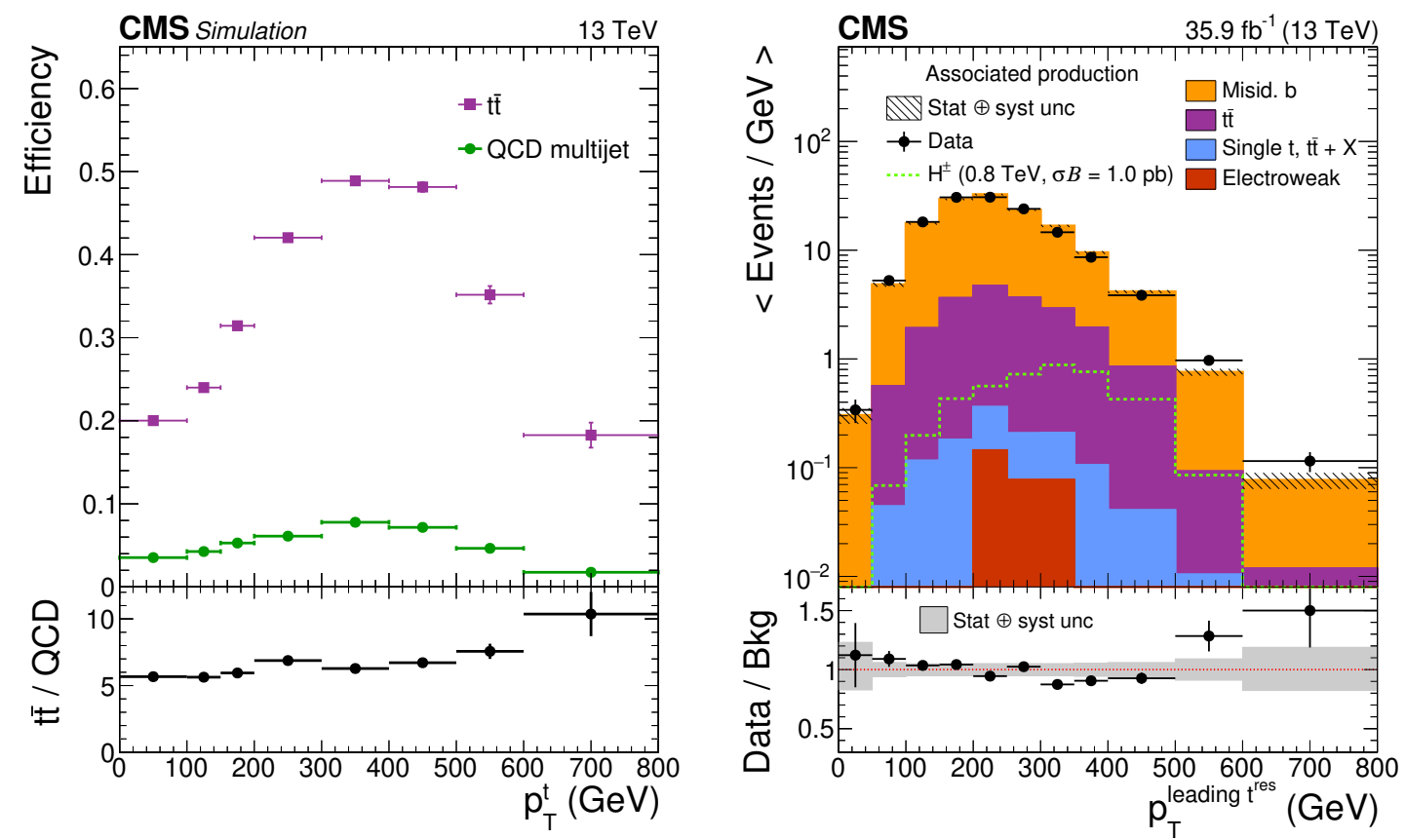

Figure 3. The efficiency of the $t^{\text {res }}$ selection in simulated $t \bar{t}$ pairs and the misidentification rate for QCD multijet background, as a function of top quark or top quark candidate $p_{\mathrm{T}}$, respectively (left). The $p_{\mathrm{T}}$ distribution of the leading $\mathrm{t}^{\text {res }}$ (right) for the signal model and background with normalization fixed to the SM expectation. The dominant background containing misidentified b jets is primarily composed of QCD multijet processes and is estimated using a data-driven method. The expectation for a signal with $m_{\mathrm{H}^{ \pm}}=0.8 \mathrm{TeV}$ is also shown.

To reconstruct the invariant mass of the $\mathrm{H}^{ \pm}$candidate, we use the resolved top quark

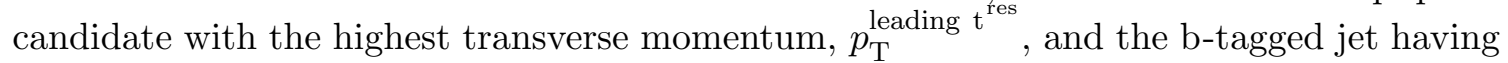
highest $p_{\mathrm{T}}$ that is not used in the reconstruction of the two selected top quark candidates. The distribution of $p_{\mathrm{T}}^{\text {leading } \mathrm{t}^{\text {res }}}$ is shown in figure 3 (right). The invariant mass $m_{\mathrm{tb}}$ of the $\mathrm{H}^{ \pm}$candidate is used in a binned maximum likelihood fit to extract the signal in the presence of the SM background.

\section{Backgrounds}

The dominant backgrounds arise from QCD multijet processes and top quark pair production in association with additional jets. Contributions from more rare processes, such as single top quark, t $\overline{\mathrm{t}}+\mathrm{X}, \mathrm{V}+\mathrm{jets}$, diboson, and triboson production are found to be small.

\subsection{Background estimations in events with boosted $W$ boson and top quark candidates}

We estimate the QCD multijet and top quark backgrounds using a method that exploits a number of background-rich control regions (CRs) in data. These control regions are included in a simultaneous fit with the signal enriched regions to determine the normalization and the shape of the background distributions. 
Because the cross section for QCD multijet production is large, this background can produce many events satisfying the signal selection requirements. The distribution of $m_{\mathrm{SD}}$ for signal peaks around the $\mathrm{W}$ boson or the top quark mass for large-radius jets corresponding to their hadronic decays, while the QCD multijet background spectrum is peaked at lower $m_{\mathrm{SD}}$. This background is estimated from simulation with corrections applied to both shape and normalization. These corrections are determined by matching the simulation to data using a $\mathrm{CR}$ enriched with jets arising from the hadronization of single quarks or gluons. The $\mathrm{CR}$ is defined by inverting the $N$-subjettiness requirements used to identify the $\mathrm{t}$ and $\mathrm{W}$ jets. The shape is determined for each event category using both this $\mathrm{CR}$ and the sideband regions around the signal mass windows in the invariant mass spectrum of the top and bottom quark pair, while the normalization is determined from the sidebands only. We validate this correction by applying the technique in an orthogonal CR defined by requiring that no b-tagged jets are identified. The shapes of the $N$-subjettiness distributions and kinematics of jets having $m_{\mathrm{SD}}$ consistent with either a $t$ or $\mathrm{W}$ jet are found to be consistent with events passing the signal selection.

The contribution from the t $\bar{t}$ process arises from all-jet final states or with a leptonic decay of a $\mathrm{W}$ boson where the charged lepton is outside the kinematic acceptance of the CMS detector or evades identification by the dedicated lepton vetoes. Such events contain a pair of $\mathrm{b}$ quarks and boosted $\mathrm{W}$ and $\mathrm{t}$ jets. The $\mathrm{t} \overline{\mathrm{t}}$ background is estimated from simulation and normalized using a CR in data. A lepton enriched set of events is used to describe the kinematics for the top quark pair production and the normalization is allowed to vary unconstrained in the final fit. The $\mathrm{CR}$ is defined by requiring a lepton $(\mathrm{e}, \mu)$ with $10<p_{\mathrm{T}}<35 \mathrm{GeV}, p_{\mathrm{T}}^{\text {miss }}>100 \mathrm{GeV}$ and at least one b-tagged jet. This ensures orthogonality with the searches for charged Higgs bosons in the leptonic channels [21].

\subsection{Background estimations in events with resolved top quarks}

The main backgrounds for the resolved analysis can be decomposed into events containing either genuine $b$ jets or events with at least one light quark or gluon jet erroneously tagged as $a b$ jet. We refer to the latter as misidentified $b$ jets. The background containing genuine $\mathrm{b}$ jets is modeled using simulation. The background due to misidentified $\mathrm{b}$ jets is measured with a data-driven technique using control regions that are defined by inverting the BDTG requirement, the $\mathrm{b}$ jet selection, or both.

The shape of the $\mathrm{H}^{ \pm}$candidate mass distribution in the background is obtained from events that are separated from the signal region (SR) by requiring that only two (of at least three) b jets pass the CSV medium working point, and the remaining jets only pass the loose CSV working point. This region is referred to as the application region (AR). In order to compensate for the different selection efficiencies between these two regions, transfer factors are used to normalize the AR to the SR. These transfer factors are determined by taking the ratio of events in two additional CRs that are orthogonal to each other and to both the AR and SR. The first CR, CR1, is obtained by requiring one $t^{\text {res }}$ candidate plus a second top quark candidate failing the BDTG requirement, and the second CR, CR2, is obtained by also altering the $\mathrm{b}$ jet selection as described above for the AR. For the regions defined as $\mathrm{AR}$ and CRs, a correction is applied to remove events containing jets from $\mathrm{b}$ quark decays 
that fail the tagging requirement. In order to minimize the effect of kinematic differences between the loose and medium working points, the background from misidentified $\mathrm{b}$ jets is evaluated separately in $p_{\mathrm{T}}$ and $\eta$ bins of the b-tagged jet used in the reconstruction of the invariant mass of the $\mathrm{H}^{ \pm}$candidate.

Because the SR and associated CRs are mutually exclusive, the expected yield of misidentified $\mathrm{b}$ jet events passing the signal selections can be predicted as:

$$
N^{\mathrm{SR}}=\sum_{i} N_{i}^{\mathrm{AR}}\left(\frac{N_{i}^{\mathrm{CR} 1}}{N_{i}^{\mathrm{CR} 2}}\right),
$$

where CR1(2) refers to the first (second) control region and the index $i$ runs over all $p_{\mathrm{T}}$ and $\eta$ bins of the aforementioned highest $p_{\mathrm{T}}$ b-tagged jet.

\section{Systematic uncertainties}

The systematic uncertainties are divided into two categories: those that affect the estimation of the background from the SM processes, and those that affect the expected signal distributions and yields.

The events used in this search are largely collected with a trigger efficiency close to $100 \%$. The trigger efficiency is extracted from data and the uncertainties in trigger correction factors applied to the simulation are less than $5 \%$.

The uncertainty from pileup modeling is estimated by varying the total inelastic pp cross section of $69.2 \mathrm{mb}$ by $5 \%$ [100]. The uncertainty in the integrated luminosity is estimated to be $2.5 \%$ [101].

Uncertainties in the background prediction that also affect the signal arise from the jet energy scale [93], from the scale factors correcting the efficiency and misidentification rate for b tagging [90], and from the reconstruction and identification efficiencies of the leptons. In addition, uncertainties arising from the simulation-to-data corrections for boosted $t$ and $\mathrm{W}$ tagging and the BDTG response are applied in the boosted and resolved analyses, respectively. The variations in the jet selection and jet energy scale are propagated to the $H_{\mathrm{T}}, p_{\mathrm{T}}^{\text {miss }}$, and $\mathrm{H}^{ \pm}$candidate yields and invariant mass.

For the boosted analysis a normalization uncertainty of $50 \%$ is applied for the QCD multijet background. This uncertainty is treated as uncorrelated among the boosted t- and $\mathrm{W}$-tagged event categories and it is $100 \%$ correlated within each category and across the signal regions and the sidebands. An additional uncertainty to account for shape variations in modeling the $H_{\mathrm{T}}$ observable is parametrized linearly as a function of $H_{\mathrm{T}}$ and reaches $30 \%$ for an $H_{\mathrm{T}}$ of $1 \mathrm{TeV}$. These uncertainties are then constrained by studying the CR used to correct the simulation and the resulting variation in expected QCD multijet background yield is approximately $28 \%$.

The systematic uncertainties affecting the misidentified $b$ jet background measurement in the resolved analysis can be divided into three components. The first component consists of events containing jets from $b$ quark decays that fail the $b$ tagging requirement and is subtracted from the CRs used in the measurement. The uncertainty on the normalization 
of this component is estimated by propagating all the uncertainties related to the simulation of electroweak and top quark processes. The other two components account for the statistical and systematic uncertainties in determining the transfer factors applied in the normalization of the AR. Statistical fluctuations in the value of the transfer factors can result in rate and shape differences in the predicted invariant mass distribution. Similarly, the definition of the $\mathrm{CR}$ affects the individual transfer factors and subsequently the invariant mass shape in the AR. The aforementioned contributions affect the expected event yield by approximately $4 \%$.

For $t \bar{t}$ and single top quark processes, the effect of the top quark mass on the cross sections is estimated by varying the top quark mass by $\pm 1.0 \mathrm{GeV}$ around the nominal value of $172.5 \mathrm{GeV}$.

Theoretical uncertainties in both the acceptance and the cross sections are determined by varying the choice of factorization and renormalization scales and PDFs. Uncertainties due to scales in the inclusive cross sections are estimated for each simulated process by varying the scales independently and together by factors of 0.5 and 2 with respect to the default values. The event yields are then calculated for each of the six variations and the maximum variation with respect to nominal is taken as the systematic uncertainty. The PDF uncertainties are treated as fully correlated for all processes that share the same dominant partons in the initial state of the matrix element calculations (i.e., gg, gq, or qq) [102].

Finally, the limited numbers of simulated background and signal events lead to statistical fluctuations in the nominal predictions. The effects are considered in the limit calculations using a Barlow-Beeston lite approach [103, 104], which assigns the combined statistical uncertainty in each bin to the overall background yield in that bin.

Tables 1 and 2 summarize the various sources of systematic uncertainty and their impact on the signal yield and the total expected background in data, for the boosted and resolved analyses, respectively.

\section{Results and interpretation}

The expected SM event yields from a background-only fit to the data are shown in figure 4 and table 3 for the boosted and resolved analyses, respectively. For the boosted analysis, the background predictions are broken down into various categories of signal- and backgroundenriched regions and in total 98 distributions are fitted. The shape of the $H_{\mathrm{T}}$ distribution in the boosted analysis and the invariant mass of the $\mathrm{H}^{ \pm}$in the resolved analysis are used to assess the agreement with the background-only hypothesis or the presence of the signal in a global binned maximum likelihood fit incorporating all the systematic uncertainties described in section 7 as nuisance parameters. The fitted distributions for the backgroundonly hypothesis are shown in figure 5 (left) for one category of the boosted analysis (t1b, $2 \mathrm{~b}, N_{\text {jets }} \geq 3$ ) and in figure 5 (right) for the resolved analysis. The contribution of a hypothetical charged Higgs boson with a mass of 0.8 or $1 \mathrm{TeV}$ and $\sigma \mathcal{B}=1 \mathrm{pb}$, assuming the associated production mechanism, is also displayed. 


\begin{tabular}{|lcccccc|}
\hline Source & Shape & $\mathrm{H}^{ \pm}$ & Multijets & $\mathrm{t} \overline{\mathrm{t}}$ & Single t, $\mathrm{t} \overline{\mathrm{t}}+\mathrm{X}$ & Electroweak \\
\hline Trigger efficiency & & 5.0 & 4.5 & 0.39 & 0.05 & 0.06 \\
Pileup & $\checkmark$ & 0.42 & 1.4 & 0.05 & $<0.01$ & 0.03 \\
Integrated luminosity & & 2.5 & - & 0.2 & 0.02 & 0.03 \\
Lepton efficiency & & 5 & - & 0.39 & 0.05 & 0.06 \\
Jet energy scale and resolution & $\checkmark$ & 3.0 & 5.8 & 0.4 & 0.04 & 0.12 \\
b jet identification & $\checkmark$ & 2.4 & 12 & 0.24 & 0.03 & 0.12 \\
Unclustered $p_{\mathrm{T}}^{\text {miss }}$ energy scale & $\checkmark$ & 0.23 & - & 0.02 & $<0.01$ & 0.01 \\
Jet $m_{\mathrm{SD}}$ scale & $\checkmark$ & 1.3 & 2.5 & 0.07 & 0.02 & - \\
$N$-subjettiness scale & & 2.0 & - & 0.17 & 0.02 & - \\
QCD bkg. normalization & & - & 28 & - & - & - \\
QCD bkg. shape & $\checkmark$ & - & $<0.01$ & - & - & - \\
Top quark mass & & - & - & 0.21 & 0.02 & - \\
Theory source & & & & & & \\
\hline Scales, PDF (acceptance) & $\checkmark$ & 2.1 & - & 0.53 & - & 0.04 \\
Scales, PDF (cross section) & & - & - & 0.43 & 0.04 & 0.05 \\
\hline
\end{tabular}

Table 1. The systematic uncertainties affecting signal and background for the boosted analysis, evaluated after fitting to data, summed over all final states and categories. The numbers are given in percentage and describe the effect of each nuisance parameter on the overall normalization of the signal model or the total background. Nuisance parameters with a check mark also affect the shape of the $H_{\mathrm{T}}$ spectrum. Sources that do not apply in a given process are marked with dashes. For the $\mathrm{H}^{ \pm}$signal, the values for $m_{\mathrm{H}^{ \pm}}=1 \mathrm{TeV}$ and for associated production are shown.

\begin{tabular}{|lcccccc|}
\hline Source & Shape & $\mathrm{H}^{ \pm}$ & Misid. b & $\mathrm{t} \overline{\mathrm{t}}$ & Single $\mathrm{t}, \mathrm{t} \overline{\mathrm{t}}+\mathrm{X}$ & Electroweak \\
\hline Trigger efficiency & & 5.0 & 0.09 & 0.69 & 0.04 & 0.01 \\
Pileup & $\checkmark$ & $<0.01$ & - & $<0.01$ & $<0.01$ & $<0.01$ \\
Integrated luminosity & & 2.5 & 0.09 & 0.35 & 0.02 & $<0.01$ \\
Lepton efficiency & & 0.32 & - & 0.04 & $<0.01$ & $<0.01$ \\
Jet energy scale and resolution & $\checkmark$ & 8.5 & 0.24 & 1.6 & 0.09 & 0.33 \\
b jet identification & $\checkmark$ & 5.0 & - & 0.64 & 0.04 & 0.01 \\
tres tagging & $\checkmark$ & 8.9 & 0.24 & 1.8 & 0.04 & $<0.01$ \\
Transfer factors & $\checkmark$ & - & 4.0 & - & - & - \\
Top quark mass & & - & 0.09 & 0.39 & 0.02 & - \\
& & & & & & 0.01 \\
Theory source & & & & & & 0.01 \\
\hline Scales, PDF (acceptance) & & 5.1 & - & 0.39 & 0.02 & \\
Scales, PDF (cross section) & & - & 0.12 & 0.76 & 0.04 & 0.01 \\
\hline
\end{tabular}

Table 2. The systematic uncertainties in the backgrounds and the signal for the resolved analysis, evaluated after fitting to data. The numbers are given in percentage and describe the effect of each nuisance parameter on the overall normalization of the signal model or the total background. Nuisance parameters with a check mark also affect the shape of the $\mathrm{H}^{ \pm}$candidate mass spectrum. Sources that do not apply in a given process are marked with dashes. For the $\mathrm{H}^{ \pm}$signal, the values for $m_{\mathrm{H}^{ \pm}}=0.5 \mathrm{TeV}$ are shown. 


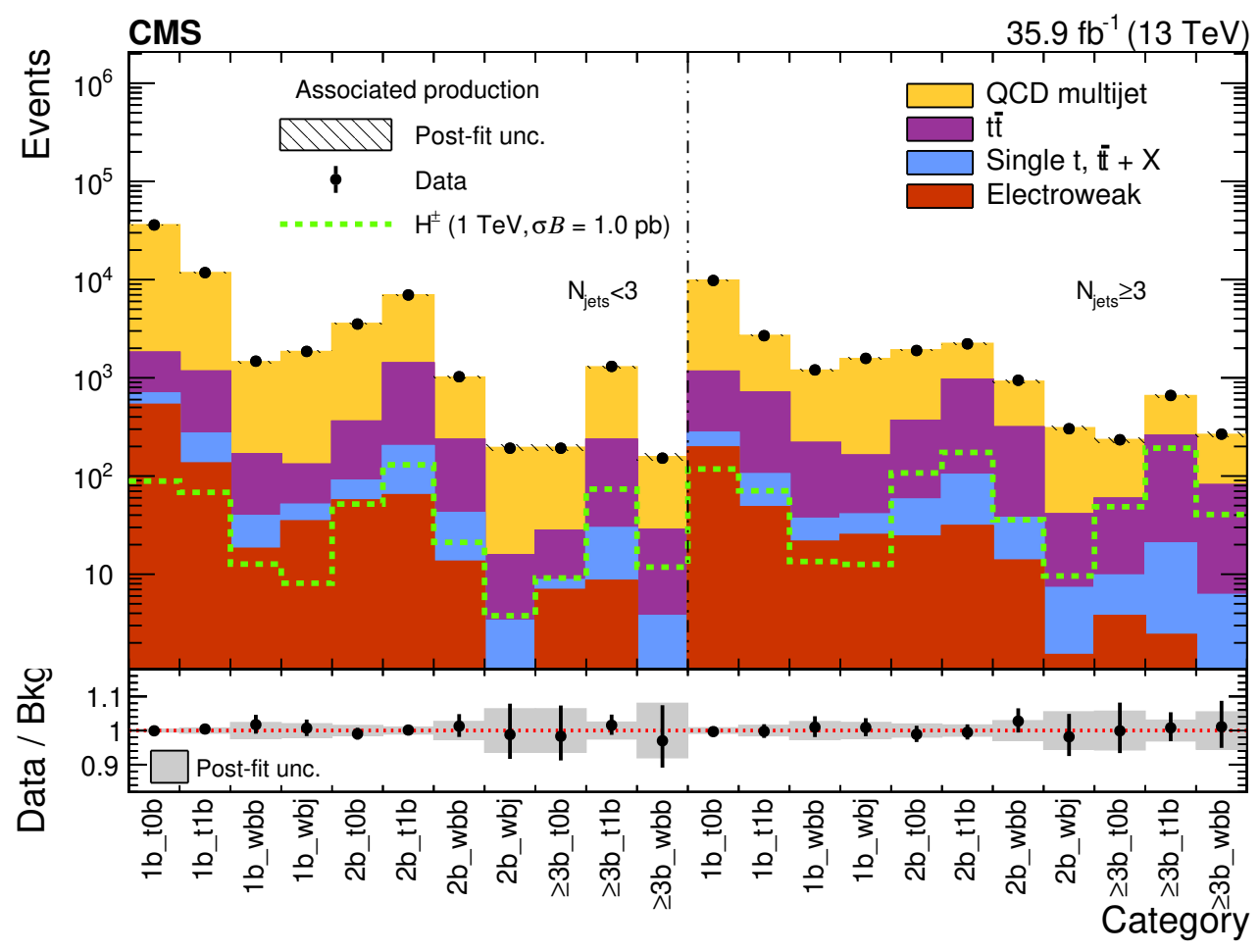

Figure 4. Expected event yields for the boosted analysis in the mass window as defined in figure 2 for an $\mathrm{H}^{ \pm}$with mass $1 \mathrm{TeV}$ in each of the signal categories used in the associated production model. The 11 categories on the left have low jet multiplicity $\left(N_{\text {jets }}<3\right)$, while categories on the right have high jet multiplicity $\left(N_{\text {jets }} \geq 3\right)$. The yields observed in data (black markers) are overlaid. The dashed lines represent the yields for an $\mathrm{H}^{ \pm}$with a mass of $1 \mathrm{TeV}$ and $\sigma \mathcal{B}=1 \mathrm{pb}$ for associated production. The background distributions result from the global fit described in the text for the background-only hypothesis. Similar categories are fitted for the $s$-channel production.

\begin{tabular}{|lc|}
\hline Process & Events \pm (stat) $\oplus$ (syst) \\
\hline Misidentified b jets & $6152 \pm 292$ \\
Genuine b jets & $1067_{-187}^{+185}$ \\
Total expected from the SM & $7220 \pm 336$ \\
Observed & 7124 \\
$\mathrm{H}^{ \pm}$signal, $m_{\mathrm{H}^{ \pm}}=0.5 \mathrm{TeV}$ & $183 \pm 26$ \\
$\mathrm{H}^{ \pm}$signal, $m_{\mathrm{H}^{ \pm}}=0.65 \mathrm{TeV}$ & $218_{-31}^{+30}$ \\
$\mathrm{H}^{ \pm}$signal, $m_{\mathrm{H}^{ \pm}}=0.8 \mathrm{TeV}$ & $234 \pm 33$ \\
\hline
\end{tabular}

Table 3. The numbers of expected and observed events for the resolved analysis after all selections. For background processes, the event yields and their corresponding uncertainties are prior to the background-only fit to the data. For the $\mathrm{H}^{ \pm}$mass hypotheses of $0.50,0.65$, and $0.80 \mathrm{TeV}$, the signal yields are normalized to a $\sigma \mathcal{B}=1 \mathrm{pb}$ and the total systematic uncertainties prior to the fit are shown. 

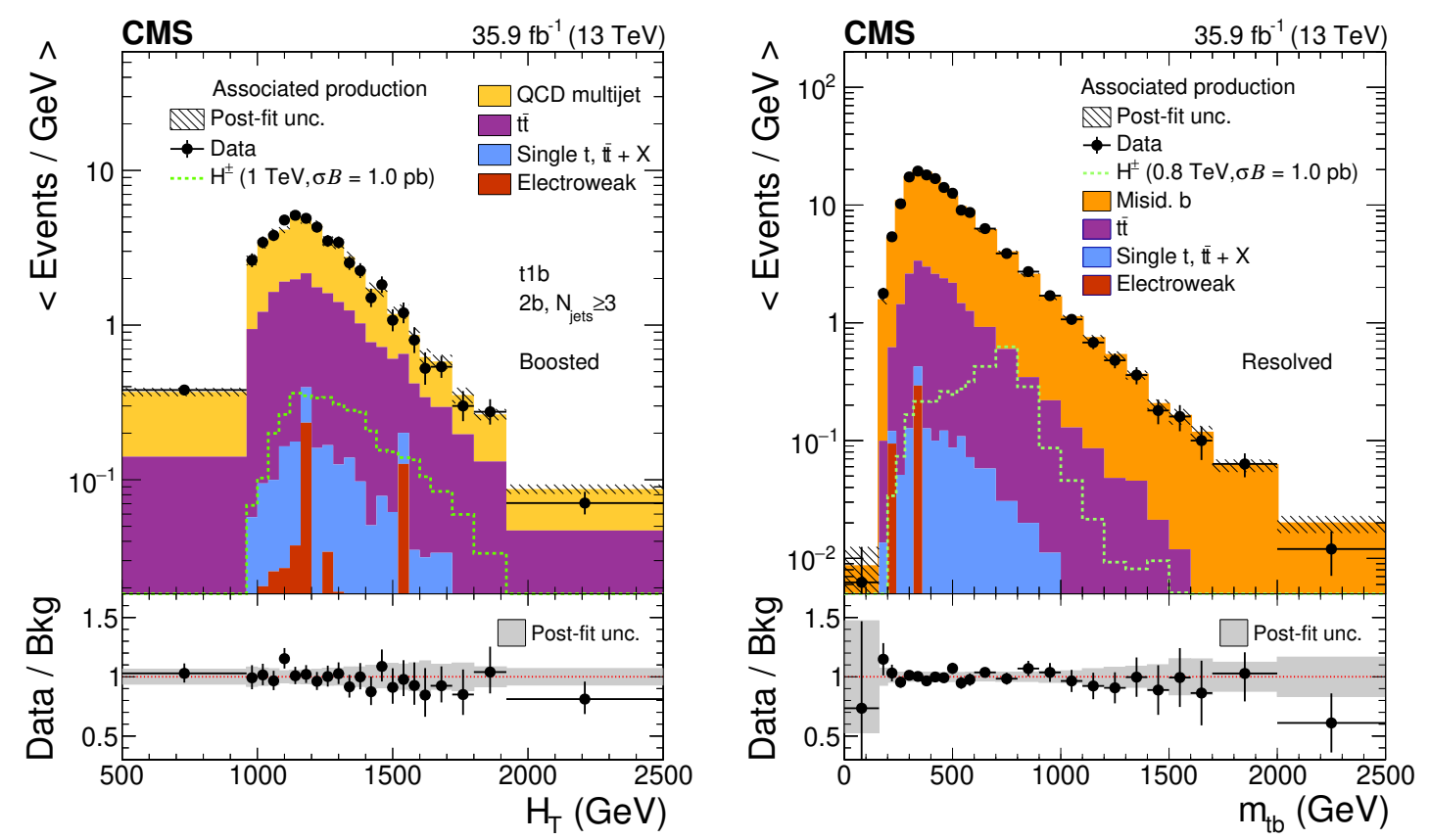

Figure 5. Variables used in the limit extraction. The $H_{\mathrm{T}}$ distribution is shown for the boosted analysis and for the category t1b, $2 \mathrm{~b}, N_{\text {jets }} \geq 3$, in the mass window (left), for the associated production channels, with the expected signal for $m_{\mathrm{H}^{ \pm}}=1 \mathrm{TeV}$. The invariant mass of the $\mathrm{H}^{ \pm}$ candidates is shown for the resolved analysis (right), with the expected signal for $m_{\mathrm{H}^{ \pm}}=0.8 \mathrm{TeV}$. The background distributions result from the background-only fit discussed in the text. The distributions are binned according to the statistical precision of the samples, leading to wider bins in the tail of the distributions.

The observed data agree with the predicted SM background processes. The results of the search are interpreted to set upper limits on the product of the charged Higgs boson production cross section and branching fraction into a top and bottom quark-antiquark pair. The upper limits are calculated at $95 \%$ confidence level (CL) using the $\mathrm{CL}_{\mathrm{s}}$ criterion $[105,106]$. An asymptotic approximation is applied for the test statistic [107, 108], $\ln \mathcal{L}_{\mu} / \mathcal{L}_{\text {max }}$, where $\mathcal{L}_{\max }$ is the maximum likelihood determined by allowing all fitted parameters, including the signal strength, $\mu$, to vary, and $\mathcal{L}_{\mu}$ is the maximum likelihood for a fixed signal strength. Results are shown for the associated production model in figure 6 (left). The reported limit at each mass value is determined by choosing the analysis strategy (resolved or boosted) with the best expected sensitivity. The data in the boosted analysis are also examined in the context of the $s$-channel model and the resulting limits are shown in figure 6 (right).

Exclusion limits are placed on the production cross section of the $\mathrm{H}^{ \pm}$associated with a top quark, $\sigma_{\mathrm{H}^{ \pm} \mathrm{t}(\mathrm{b})} \mathcal{B}\left(\mathrm{H}^{ \pm} \rightarrow \mathrm{tb}\right)=\sigma_{\mathrm{pp} \rightarrow \mathrm{H}^{+} \overline{\mathrm{t}}(\mathrm{b})} \mathcal{B}\left(\mathrm{H}^{+} \rightarrow \mathrm{t} \overline{\mathrm{b}}\right)+\sigma_{\mathrm{pp} \rightarrow \mathrm{H}^{-} \mathrm{t}(\overline{\mathrm{b}})} \mathcal{B}\left(\mathrm{H}^{-} \rightarrow \overline{\mathrm{tb}}\right)$, for masses from 0.2 to $3 \mathrm{TeV}$ in the range 21.3 to $0.007 \mathrm{pb}$. The boosted analysis has the best sensitivity for $m_{\mathrm{H}^{ \pm}}$larger than $0.8 \mathrm{TeV}$ while the resolved analysis limits are most stringent at lower masses. The boosted analysis sets upper limits from 4.5 to $0.023 \mathrm{pb}$ on the $\mathrm{H}^{ \pm}$production cross section in the $s$-channel, $\sigma\left(\mathrm{pp} \rightarrow \mathrm{H}^{ \pm}\right) \mathcal{B}\left(\mathrm{H}^{ \pm} \rightarrow \mathrm{tb}\right)$, for masses from 0.8 to $3 \mathrm{TeV}$, extending the regions excluded from prior results [19]. 

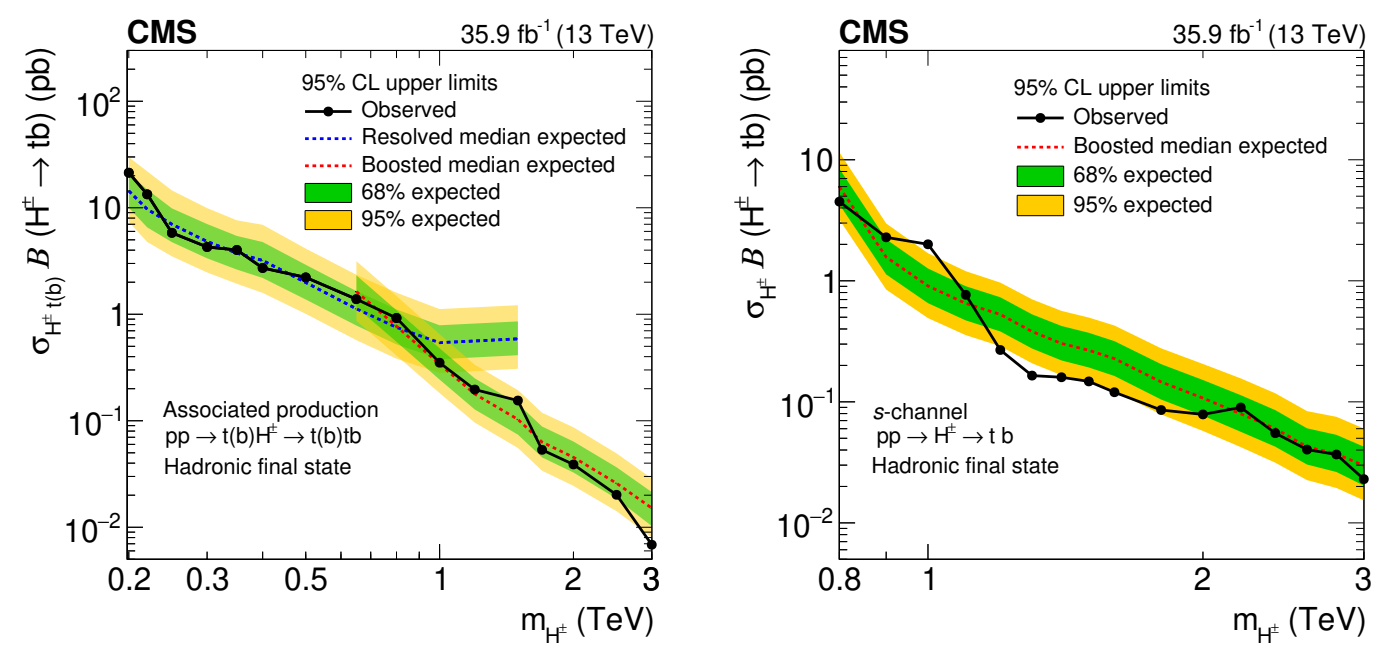

Figure 6. Upper limits at 95\% CL on the product of the $\mathrm{H}^{ \pm}$production cross section and branching fraction as a function of $m_{\mathrm{H}^{ \pm}}$for the associated (left) and $s$-channel (right) processes. The observed upper limits are shown by the solid black markers. The median expected limit (dashed line), 68\% (inner green band), and 95\% (outer yellow band) confidence interval for the expected limits are also shown. For the association production model limits are calculated from the resolved (boosted) analysis for $m_{\mathrm{H}^{ \pm}}$points up to (greater than) $0.8 \mathrm{TeV}$.

Model-dependent upper limits are obtained by comparing the observed limit in the association production model with theoretical predictions provided by the LHC-HXSWG [16]. The hMSSM benchmark scenario [109-112] assumes that the discovered Higgs boson is the light Higgs boson in the 2HDM and that the SUSY particles have masses too large to be directly observed at the LHC. The $M_{\mathrm{h}}^{125}(\widetilde{\mathrm{X}})$ scenario [113] is characterized as having significant mixing between higgsinos and gauginos, and a compressed mass spectrum of charginos and neutralinos. Its phenomenology differs from the Type II $2 \mathrm{HDM}$ due to the presence of light charginos and neutralinos, such that heavy Higgs bosons are allowed to decay to these superpartners. Higgs masses and mixing in the $M_{\mathrm{h}}^{125}(\widetilde{\chi})$ scenario are computed by FeynHiggs $[45,114-119]$ in each point of the $\left(m_{\mathrm{A}}, \tan \beta\right)$ plane. The branching fractions in the hMSSM scenario are calculated with $\operatorname{HDECAY}[51,120,121]$ alone, while the $M_{\mathrm{h}}^{125}(\widetilde{\mathrm{\chi}})$ scenario combines the most precise results of FEYNHigGs, HDECAY and PROPHECY4F $[122,123]$. Figure 7 shows the excluded parameter space in these MSSM scenarios. In the hMSSM scenario the maximum $\tan \beta$ value excluded is 0.88 for $m_{\mathrm{H}^{ \pm}}$values between 0.20 and $0.55 \mathrm{TeV}$. In the $M_{\mathrm{h}}^{125}(\widetilde{\mathrm{x}})$ scenario the maximum $\tan \beta$ value excluded is 0.86 for $m_{\mathrm{H}^{ \pm}}$values between 0.20 and $0.57 \mathrm{TeV}$.

\section{Combination with the leptonic final states}

In ref. [21] a search is presented for an $\mathrm{H}^{ \pm}$with mass greater than that of the top quark and decaying into a top and bottom quark-antiquark pair in the complementary leptonic final states. Events are selected by the presence of a single isolated charged lepton (e or $\mu$ ) or an opposite-sign dilepton pair $(\mathrm{ee}, \mu \mu, \mathrm{e} \mu)$. These are categorized according to the jet 

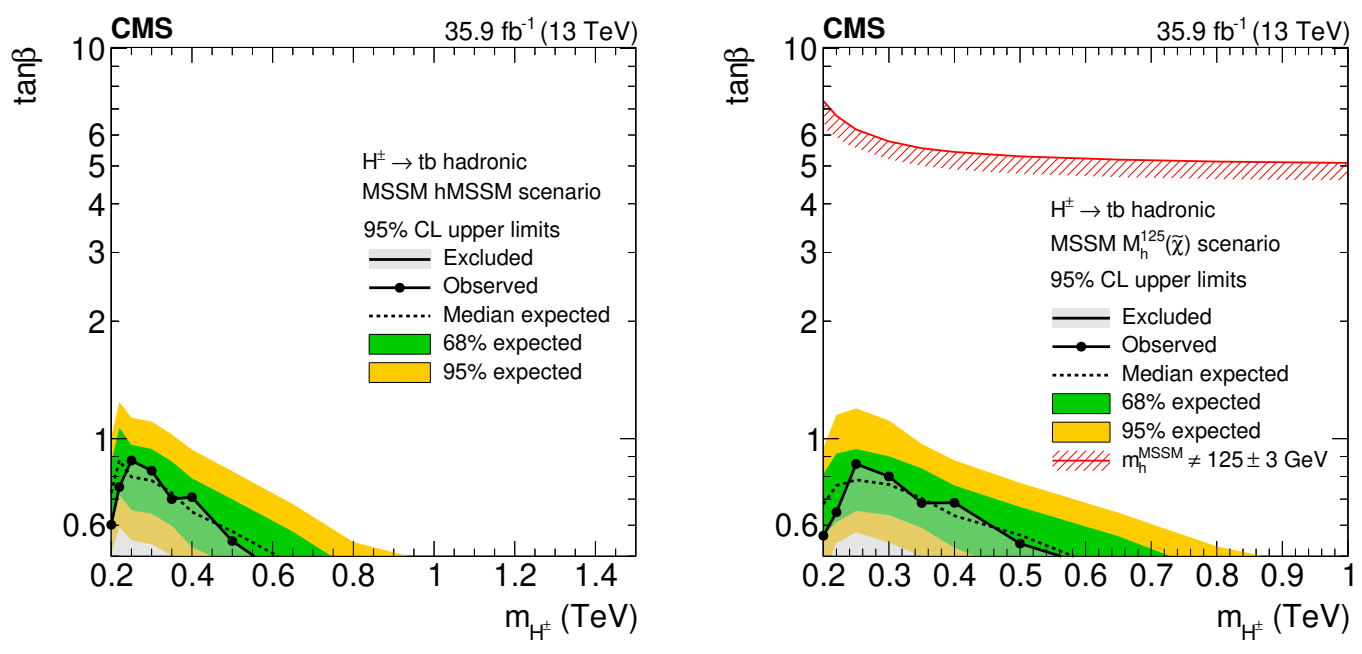

Figure 7. Excluded parameter space region in the hMSSM scenario (left) and $M_{\mathrm{h}}^{125}(\widetilde{\mathrm{\chi}})$ (right) using the association production model. The observed upper limits are shown by the solid black markers. The median expected limit (dashed line), 68\% (inner green band), and 95\% (outer yellow band) confidence interval for the expected limits are also shown. The region below the red line is excluded assuming that the observed neutral Higgs boson is the light CP-even 2HDM Higgs boson with a mass of $125 \pm 3 \mathrm{GeV}$, where the uncertainty is the theoretical uncertainty in the mass calculation.

multiplicity and number of b-tagged jets and multivariate techniques are used to enhance the signal and background discrimination in each category. The search is based on the same pp collision data collected by the CMS experiment at a center-of-mass energy of $13 \mathrm{TeV}$, corresponding to an integrated luminosity of $35.9 \mathrm{fb}^{-1}$.

These results are combined with those from the all-jet channel analyses to calculate the $95 \%$ CL combined upper limits on the product of the cross section and the branching fraction as a function of the $m_{\mathrm{H}^{ \pm}}$for the process $\sigma_{\mathrm{H}^{ \pm} \mathrm{t}(\mathrm{b})} \mathcal{B}\left(\mathrm{H}^{ \pm} \rightarrow \mathrm{tb}\right)$. The limits are shown in figure 8 and table 4 . The common experimental and theoretical nuisance parameters between final states sharing the same production mechanism are correlated, while the uncertainties from different sources described in section 7 are assumed to be uncorrelated. The single-lepton final state has the best sensitivity in the whole $m_{\mathrm{H}^{ \pm}}$range from 0.2 to $3 \mathrm{TeV}$, while the dilepton channel contributes in the low $m_{\mathrm{H}^{ \pm}}$regime, i.e., $\leq 1.5 \mathrm{TeV}$, and the all-jet channel improves the overall sensitivity by $20-25 \%$ at larger values of $m_{\mathrm{H}^{ \pm}}$.

\section{Summary}

Results are presented from a search for charged Higgs bosons $\left(\mathrm{H}^{ \pm}\right)$that decay to a top and a bottom quark in the all-jet final state. The search considers two distinct event topologies. The $\mathrm{H}^{ \pm}$is reconstructed from a b-tagged jet in combination with a top quark candidate, either resolved as two jets from $\mathrm{q}^{\prime}{ }^{\prime}$ decays of a $\mathrm{W}$ boson and an additional b-tagged jet, or, for highly boosted decay products, reconstructed as a single top-flavored jet or a $\mathrm{W}$ jet paired with an additional b-tagged jet. The analysis uses data collected with 

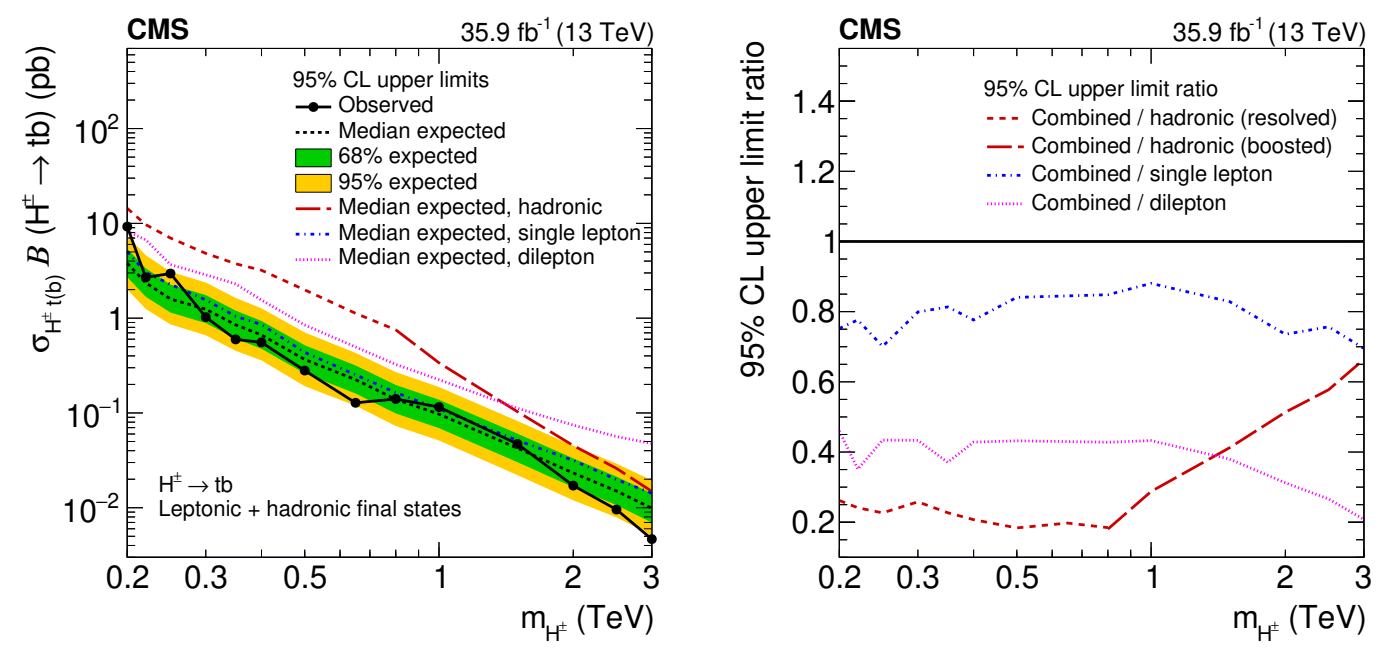

Figure 8. Upper limits at 95\% CL on the product of the $\mathrm{H}^{ \pm}$production cross section and branching fraction as a function of $m_{\mathrm{H}^{ \pm}}$for the process $\sigma_{\mathrm{H}^{ \pm} \mathrm{t}(\mathrm{b})} \mathcal{B}\left(\mathrm{H}^{ \pm} \rightarrow \mathrm{tb}\right)$. The median expected limit (dashed line), $68 \%$ (inner green band), and 95\% (outer yellow band) confidence interval expected limits are also shown (left). The relative expected contributions of each channel to the overall combination are shown (right). The black solid line corresponds to the combined expected limits while the dashed, dotted and dash-dotted lines represent the contributing channels.

\begin{tabular}{|c|c|c|c|c|c|c|}
\hline \multirow[b]{2}{*}{$m_{\mathrm{H}^{ \pm}}(\mathrm{TeV})$} & \multicolumn{5}{|c|}{ Expected limits (pb) } & \multirow[t]{2}{*}{ Observed limits (pb) } \\
\hline & -2 s.d. & -1 s.d. & median & +1 s.d. & +2 s.d. & \\
\hline 0.20 & 2.02 & 2.71 & 3.80 & 5.39 & 7.38 & 9.25 \\
\hline 0.22 & 1.25 & 1.69 & 2.36 & 3.36 & 4.62 & 2.69 \\
\hline 0.25 & 0.86 & 1.15 & 1.59 & 2.26 & 3.09 & 2.96 \\
\hline 0.30 & 0.66 & 0.89 & 1.24 & 1.75 & 2.38 & 1.03 \\
\hline 0.35 & 0.45 & 0.61 & 0.85 & 1.20 & 1.64 & 0.60 \\
\hline 0.40 & 0.36 & 0.48 & 0.66 & 0.93 & 1.27 & 0.56 \\
\hline 0.50 & 0.19 & 0.26 & 0.37 & 0.52 & 0.71 & 0.28 \\
\hline 0.65 & 0.12 & 0.16 & 0.22 & 0.32 & 0.43 & 0.13 \\
\hline 0.80 & 0.073 & 0.099 & 0.14 & 0.20 & 0.27 & 0.14 \\
\hline 1.00 & 0.051 & 0.069 & 0.097 & 0.14 & 0.19 & 0.12 \\
\hline 1.50 & 0.022 & 0.030 & 0.043 & 0.060 & 0.082 & 0.047 \\
\hline 2.00 & 0.012 & 0.017 & 0.023 & 0.033 & 0.045 & 0.017 \\
\hline 2.50 & 0.008 & 0.011 & 0.015 & 0.021 & 0.029 & 0.010 \\
\hline 3.00 & 0.005 & 0.007 & 0.010 & 0.014 & 0.019 & 0.005 \\
\hline
\end{tabular}

Table 4. The upper limit at 95\% CL on $\sigma_{\mathrm{H}^{ \pm} \mathrm{t}(\mathrm{b})} \mathcal{B}\left(\mathrm{H}^{ \pm} \rightarrow \mathrm{tb}\right)$ with the combined all-jet, singlelepton, and dilepton final states.

the CMS detector in 2016 at a center-of-mass energy of $\sqrt{s}=13 \mathrm{TeV}$, corresponding to an integrated luminosity of $35.9 \mathrm{fb}^{-1}$. No significant deviation is observed above the expected standard model background. Model-independent upper limits at $95 \%$ confidence level are set on the product of the $\mathrm{H}^{ \pm}$production cross section and its branching fraction into a 
top and bottom quark-antiquark pair. For production in association with a top quark, limits of 21.3 to $0.007 \mathrm{pb}$ are set for $\mathrm{H}^{ \pm}$masses in the range 0.2 to $3 \mathrm{TeV}$. Combining these results with those from a search in leptonic final states of $\mathrm{W}$ bosons sets improved limits of 9.25 to $0.005 \mathrm{pb}$. Exclusion regions are also presented in the parameter space of the minimal supersymmetric standard model hMSSM and $M_{\mathrm{h}}^{125}(\widetilde{\chi})$ benchmark scenarios. The complementary $s$-channel production of an $\mathrm{H}^{ \pm}$is investigated in the mass range 0.8 to $3 \mathrm{TeV}$ and the corresponding upper limits are set at 4.5 to $0.023 \mathrm{pb}$.

\section{Acknowledgments}

We congratulate our colleagues in the CERN accelerator departments for the excellent performance of the LHC and thank the technical and administrative staffs at CERN and at other CMS institutes for their contributions to the success of the CMS effort. In addition, we gratefully acknowledge the computing centers and personnel of the Worldwide LHC Computing Grid for delivering so effectively the computing infrastructure essential to our analyses. Finally, we acknowledge the enduring support for the construction and operation of the LHC and the CMS detector provided by the following funding agencies: BMBWF and FWF (Austria); FNRS and FWO (Belgium); CNPq, CAPES, FAPERJ, FAPERGS, and FAPESP (Brazil); MES (Bulgaria); CERN; CAS, MoST, and NSFC (China); COLCIENCIAS (Colombia); MSES and CSF (Croatia); RPF (Cyprus); SENESCYT (Ecuador); MoER, ERC IUT, PUT and ERDF (Estonia); Academy of Finland, MEC, and HIP (Finland); CEA and CNRS/IN2P3 (France); BMBF, DFG, and HGF (Germany); GSRT (Greece); NKFIA (Hungary); DAE and DST (India); IPM (Iran); SFI (Ireland); INFN (Italy); MSIP and NRF (Republic of Korea); MES (Latvia); LAS (Lithuania); MOE and UM (Malaysia); BUAP, CINVESTAV, CONACYT, LNS, SEP, and UASLP-FAI (Mexico); MOS (Montenegro); MBIE (New Zealand); PAEC (Pakistan); MSHE and NSC (Poland); FCT (Portugal); JINR (Dubna); MON, RosAtom, RAS, RFBR, and NRC KI (Russia); MESTD (Serbia); SEIDI, CPAN, PCTI, and FEDER (Spain); MOSTR (Sri Lanka); Swiss Funding Agencies (Switzerland); MST (Taipei); ThEPCenter, IPST, STAR, and NSTDA (Thailand); TUBITAK and TAEK (Turkey); NASU (Ukraine); STFC (United Kingdom); DOE and NSF (U.S.A.).

Individuals have received support from the Marie-Curie program and the European Research Council and Horizon 2020 Grant, contract Nos. 675440, 752730, and 765710 (European Union); the Leventis Foundation; the A.P. Sloan Foundation; the Alexander von Humboldt Foundation; the Belgian Federal Science Policy Office; the Fonds pour la Formation à la Recherche dans l'Industrie et dans l'Agriculture (FRIA-Belgium); the Agentschap voor Innovatie door Wetenschap en Technologie (IWT-Belgium); the F.R.S.-FNRS and FWO (Belgium) under the "Excellence of Science - EOS" — be.h project n. 30820817; the Beijing Municipal Science \& Technology Commission, No. Z191100007219010; the Ministry of Education, Youth and Sports (MEYS) of the Czech Republic; the Deutsche Forschungsgemeinschaft (DFG) under Germany's Excellence Strategy — EXC 2121 "Quantum Universe" - 390833306; the Lendület ("Momentum") Program and the János Bolyai Research Scholarship of the Hungarian Academy of Sciences, the New National Excellence 
Program ÚNKP, the NKFIA research grants 123842, 123959, 124845, 124850, 125105, 128713, 128786, and 129058 (Hungary); the Council of Science and Industrial Research, India; the HOMING PLUS program of the Foundation for Polish Science, cofinanced from European Union, Regional Development Fund, the Mobility Plus program of the Ministry of Science and Higher Education, the National Science Center (Poland), contracts Harmonia 2014/14/M/ST2/00428, Opus 2014/13/B/ST2/02543, 2014/15/B/ST2/03998, and 2015/19/B/ST2/02861, Sonata-bis 2012/07/E/ST2/01406; the National Priorities Research Program by Qatar National Research Fund; the Ministry of Science and Education, grant no. 14.W03.31.0026 (Russia); the Programa Estatal de Fomento de la Investigación Científica y Técnica de Excelencia María de Maeztu, grant MDM-2015-0509 and the Programa Severo Ochoa del Principado de Asturias; the Thalis and Aristeia programs cofinanced by EU-ESF and the Greek NSRF; the Rachadapisek Sompot Fund for Postdoctoral Fellowship, Chulalongkorn University and the Chulalongkorn Academic into Its 2nd Century Project Advancement Project (Thailand); the Kavli Foundation; the Nvidia Corporation; the SuperMicro Corporation; the Welch Foundation, contract C-1845; and the Weston Havens Foundation (U.S.A.).

Open Access. This article is distributed under the terms of the Creative Commons Attribution License (CC-BY 4.0), which permits any use, distribution and reproduction in any medium, provided the original author(s) and source are credited.

\section{References}

[1] ATLAS collaboration, Observation of a new particle in the search for the Standard Model Higgs boson with the ATLAS detector at the LHC, Phys. Lett. B 716 (2012) 1 [arXiv:1207.7214] [INSPIRE].

[2] CMS collaboration, Observation of a new boson at a mass of $125 \mathrm{GeV}$ with the CMS experiment at the LHC, Phys. Lett. B 716 (2012) 30 [arXiv:1207.7235] [INSPIRE].

[3] CMS collaboration, Observation of a new boson with mass near $125 \mathrm{GeV}$ in pp Collisions at $\sqrt{s}=7$ and $8 \mathrm{TeV}$, JHEP 06 (2013) 081 [arXiv:1303.4571] [INSPIRE].

[4] ATLAS and CMS collaborations, Combined measurement of the Higgs boson mass in pp collisions at $\sqrt{s}=7$ and $8 \mathrm{TeV}$ with the ATLAS and CMS experiments, Phys. Rev. Lett. 114 (2015) 191803 [arXiv:1503.07589] [INSPIRE].

[5] CMS collaboration, Measurements of properties of the Higgs boson decaying into the four-lepton final state in pp collisions at $\sqrt{s}=13 \mathrm{TeV}$, JHEP 11 (2017) 047 [arXiv:1706.09936] [INSPIRE].

[6] CMS collaboration, Constraints on the spin-parity and anomalous HVV couplings of the Higgs boson in proton collisions at 7 and 8 TeV, Phys. Rev. D 92 (2015) 012004 [arXiv: 1411.3441] [INSPIRE].

[7] ATLAS collaboration, Study of the spin and parity of the Higgs boson in diboson decays with the ATLAS detector, Eur. Phys. J. C 75 (2015) 476 [Erratum ibid. 76 (2016) 152] [arXiv: 1506. 05669] [INSPIRE].

[8] ATLAS, CMS collaboration, Measurements of the Higgs boson production and decay rates and constraints on its couplings from a combined ATLAS and CMS analysis of the LHC pp collision data at $\sqrt{s}=7$ and 8 TeV, JHEP 08 (2016) 045 [arXiv:1606.02266] [INSPIRE]. 
[9] J.F. Gunion and H.E. Haber, The CP conserving two Higgs doublet model: the approach to the decoupling limit, Phys. Rev. D 67 (2003) 075019 [hep-ph/0207010] [INSPIRE].

[10] A.G. Akeroyd et al., Prospects for charged Higgs searches at the LHC, Eur. Phys. J. C 77 (2017) 276 [arXiv: 1607.01320] [InSPIRE].

[11] G.C. Branco et al., Theory and phenomenology of two-Higgs-doublet models, Phys. Rept. 516 (2012) 1 [arXiv: 1106.0034] [INSPIRE].

[12] N. Craig and S. Thomas, Exclusive signals of an extended Higgs sector, JHEP 11 (2012) 083 [arXiv: 1207.4835] [INSPIRE].

[13] A. Djouadi, The anatomy of electro-weak symmetry breaking. II. The Higgs bosons in the minimal supersymmetric model, Phys. Rept. 459 (2008) 1 [hep-ph/0503173] [INSPIRE].

[14] M. Carena, S. Heinemeyer, O. Stål, C.E.M. Wagner and G. Weiglein, MSSM Higgs boson searches at the LHC: benchmark scenarios after the discovery of a Higgs-like particle, Eur. Phys. J. C $\mathbf{7 3}$ (2013) 2552 [arXiv: 1302.7033] [INSPIRE].

[15] M. Carena, I. Low, N.R. Shah and C.E.M. Wagner, Impersonating the standard model Higgs boson: alignment without decoupling, JHEP 04 (2014) 015 [arXiv:1310.2248] [INSPIRE].

[16] LHC Higgs Cross Section Working Group collaboration, Handbook of LHC Higgs Cross Sections: 4. Deciphering the nature of the Higgs Sector, arXiv:1610.07922 [INSPIRE].

[17] R. Harlander, M. Krämer and M. Schumacher, Bottom-quark associated Higgs-boson production: reconciling the four- and five-flavour scheme approach, arXiv:1112.3478 [INSPIRE].

[18] CMS collaboration, Search for a charged Higgs boson in pp collisions at $\sqrt{s}=8$ TeV, JHEP 11 (2015) 018 [arXiv: 1508.07774] [INSPIRE].

[19] ATLAS collaboration, Search for charged Higgs bosons in the $H^{ \pm} \rightarrow t b$ decay channel in $p p$ collisions at $\sqrt{s}=8 \mathrm{TeV}$ using the ATLAS detector, JHEP 03 (2016) 127 [arXiv: 1512.03704] [INSPIRE].

[20] ATLAS collaboration, Search for charged Higgs bosons decaying into top and bottom quarks at $\sqrt{s}=13$ TeV with the ATLAS detector, JHEP 11 (2018) 085 [arXiv:1808.03599] [INSPIRE].

[21] CMS collaboration, Search for a charged Higgs boson decaying into top and bottom quarks in events with electrons or muons in proton-proton collisions at $\sqrt{s}=13 \mathrm{TeV}$, JHEP 01 (2020) 096 [arXiv: 1908.09206] [inSPIRE].

[22] CMS collaboration, Search for a light charged Higgs boson decaying to $c \bar{s}$ in pp collisions at $\sqrt{s}=8 \mathrm{TeV}$, JHEP 12 (2015) 178 [arXiv:1510.04252] [INSPIRE].

[23] CMS collaboration, Search for a charged Higgs boson decaying to charm and bottom quarks in proton-proton collisions at $\sqrt{s}=8$ TeV, JHEP 11 (2018) 115 [arXiv:1808.06575] [INSPIRE].

[24] ATLAS collaboration, Search for charged Higgs bosons decaying via $H^{ \pm} \rightarrow \tau^{ \pm} \nu$ in fully hadronic final states using pp collision data at $\sqrt{s}=8 \mathrm{TeV}$ with the ATLAS detector, JHEP 03 (2015) 088 [arXiv: 1412.6663] [INSPIRE].

[25] ATLAS collaboration, Search for charged Higgs bosons decaying via $H^{ \pm} \rightarrow \tau^{ \pm} \nu_{\tau}$ in the $\tau+$ jets and $\tau+$ lepton final states with $36 \mathrm{fb}^{-1}$ of $p p$ collision data recorded at $\sqrt{\mathrm{s}}=13 \mathrm{TeV}$ with the ATLAS experiment, JHEP 09 (2018) 139 [arXiv: 1807.07915] [INSPIRE]. 
[26] CMS collaboration, Search for charged Higgs bosons in the $H^{ \pm} \rightarrow \tau^{ \pm} \nu_{\tau}$ decay channel in proton-proton collisions at $\sqrt{s}=13 \mathrm{TeV}$, JHEP 07 (2019) 142 [arXiv:1903.04560] [INSPIRE].

[27] BaBar, Belle collaboration, The physics of the B factories, Eur. Phys. J. C 74 (2014) 3026 [arXiv: 1406.6311] [INSPIRE].

[28] BeLle-II collaboration, The Belle II physics book, PTEP 2019 (2019) 123C01 [Erratum ibid. 2020 (2020) 029201] [arXiv: 1808.10567] [INSPIRE].

[29] CMS collaboration, Search for beyond the standard model Higgs bosons decaying into a $b \bar{b}$ pair in pp collisions at $\sqrt{s}=13 \mathrm{TeV}$, JHEP 08 (2018) 113 [arXiv: 1805.12191] [inSPIRE].

[30] CMS collaboration, Search for additional neutral MSSM Higgs bosons in the $\tau \tau$ final state in proton-proton collisions at $\sqrt{s}=13 \mathrm{TeV}$, JHEP 09 (2018) 007 [arXiv: 1803.06553] [INSPIRE].

[31] ATLAS collaboration, Search for additional heavy neutral Higgs and gauge bosons in the ditau final state produced in $36 \mathrm{fb}^{-1}$ of $\mathrm{pp}$ collisions at $\sqrt{\mathrm{s}}=13 \mathrm{TeV}$ with the ATLAS detector, JHEP 01 (2018) 055 [arXiv:1709.07242] [INSPIRE].

[32] ATLAS collaboration, Search for heavy Higgs bosons $A / H$ decaying to a top quark pair in pp collisions at $\sqrt{s}=8 \mathrm{TeV}$ with the ATLAS detector, Phys. Rev. Lett. 119 (2017) 191803 [arXiv: 1707.06025] [INSPIRE].

[33] CMS collaboration, Search for physics beyond the standard model in events with two leptons of same sign, missing transverse momentum and jets in proton-proton collisions at $\sqrt{s}=13$ TeV, Eur. Phys. J. C 77 (2017) 578 [arXiv: 1704.07323] [InSPIRE].

[34] CMS collaboration, Search for heavy Higgs bosons decaying to a top quark pair in proton-proton collisions at $\sqrt{s}=13 \mathrm{TeV}$, JHEP 04 (2020) 171 [arXiv:1908.01115] [INSPIRE].

[35] ATLAS collaboration, Search for heavy neutral Higgs bosons produced in association with b-quarks and decaying to b-quarks at $\sqrt{s}=13 \mathrm{TeV}$ with the ATLAS detector, arXiv: 1907.02749 [INSPIRE].

[36] H. Georgi and M. Machacek, Doubly charged Higgs bosons, Nucl. Phys. B 262 (1985) 463 [INSPIRE].

[37] ATLAS collaboration, Search for a charged Higgs boson produced in the vector-boson fusion mode with Decay $H^{ \pm} \rightarrow W^{ \pm} Z$ using pp collisions at $\sqrt{s}=8 \mathrm{TeV}$ with the ATLAS experiment, Phys. Rev. Lett. 114 (2015) 231801 [arXiv:1503.04233] [INSPIRE].

[38] CMS collaboration, Search for charged Higgs bosons produced via vector boson fusion and decaying into a pair of $W$ and $Z$ bosons using pp collisions at $\sqrt{s}=13$ TeV, Phys. Rev. Lett. 119 (2017) 141802 [arXiv:1705.02942] [INSPIRE].

[39] CMS collaboration, Observation of electroweak production of same-sign $W$ boson pairs in the two jet and two same-sign lepton final state in proton-proton collisions at $\sqrt{s}=13 \mathrm{TeV}$, Phys. Rev. Lett. 120 (2018) 081801 [arXiv:1709.05822] [INSPIRE].

[40] E. Malkawi, T.M.P. Tait and C.P. Yuan, A model of strong flavor dynamics for the top quark, Phys. Lett. B 385 (1996) 304 [hep-ph/9603349] [INSPIRE].

[41] CMS collaboration, Search for heavy resonances decaying to a top quark and a bottom quark in the lepton+jets final state in proton-proton collisions at $13 \mathrm{TeV}$, Phys. Lett. B 777 (2018) 39 [arXiv:1708.08539] [INSPIRE]. 
[42] CMS collaboration, The CMS trigger system, 2017 JINST 12 P01020 [arXiv:1609. 02366] [INSPIRE].

[43] CMS collaboration, The CMS experiment at the CERN LHC, 2008 JINST 3 S08004 [INSPIRE].

[44] J. Alwall et al., The automated computation of tree-level and next-to-leading order differential cross sections and their matching to parton shower simulations, JHEP $\mathbf{0 7}$ (2014) 079 [arXiv: 1405.0301] [INSPIRE].

[45] S. Heinemeyer, W. Hollik and G. Weiglein, FeynHiggs: a program for the calculation of the masses of the neutral CP even Higgs bosons in the MSSM, Comput. Phys. Commun. 124 (2000) 76 [hep-ph/9812320] [INSPIRE].

[46] E.L. Berger, T. Han, J. Jiang and T. Plehn, Associated production of a top quark and a charged Higgs boson, Phys. Rev. D 71 (2005) 115012 [hep-ph/0312286] [INSPIRE].

[47] M. Flechl et al., Improved cross-section predictions for heavy charged Higgs boson production at the LHC, Phys. Rev. D 91 (2015) 075015 [arXiv:1409.5615] [INSPIRE].

[48] C. Degrande, M. Ubiali, M. Wiesemann and M. Zaro, Heavy charged Higgs boson production at the LHC, JHEP 10 (2015) 145 [arXiv:1507.02549] [INSPIRE].

[49] S. Dittmaier, M. Krämer, M. Spira and M. Walser, Charged-Higgs-boson production at the LHC: NLO supersymmetric QCD corrections, Phys. Rev. D 83 (2011) 055005 [arXiv:0906.2648] [INSPIRE].

[50] CoMPHEP collaboration, CompHEP 4.4: automatic computations from Lagrangians to events, Nucl. Instrum. Meth. A 534 (2004) 250 [hep-ph/0403113] [INSPIRE].

[51] A. Djouadi, J. Kalinowski and M. Spira, HDECAY: a program for Higgs boson decays in the standard model and its supersymmetric extension, Comput. Phys. Commun. 108 (1998) 56 [hep-ph/9704448] [inSPIRE].

[52] P. Nason, A new method for combining NLO QCD with shower Monte Carlo algorithms, JHEP 11 (2004) 040 [hep-ph/0409146] [INSPIRE].

[53] S. Frixione, P. Nason and C. Oleari, Matching NLO QCD computations with Parton Shower simulations: the POWHEG method, JHEP 11 (2007) 070 [arXiv: 0709. 2092] [INSPIRE].

[54] S. Alioli, P. Nason, C. Oleari and E. Re, A general framework for implementing NLO calculations in shower Monte Carlo programs: the POWHEG BOX, JHEP 06 (2010) 043 [arXiv: 1002.2581] [INSPIRE].

[55] S. Frixione, P. Nason and G. Ridolfi, A positive-weight next-to-leading-order Monte Carlo for heavy flavour hadroproduction, JHEP 09 (2007) 126 [arXiv:0707.3088] [INSPIRE].

[56] R. Frederix, E. Re and P. Torrielli, Single-top t-channel hadroproduction in the four-flavour scheme with POWHEG and aMC@NLO, JHEP 09 (2012) 130 [arXiv:1207.5391] [INSPIRE].

[57] P. Artoisenet, R. Frederix, O. Mattelaer and R. Rietkerk, Automatic spin-entangled decays of heavy resonances in Monte Carlo simulations, JHEP 03 (2013) 015 [arXiv:1212.3460] [INSPIRE].

[58] E. Re, Single-top Wt-channel production matched with parton showers using the POWHEG method, Eur. Phys. J. C 71 (2011) 1547 [arXiv:1009.2450] [INSPIRE].

[59] S. Frixione et al., Single-top hadroproduction in association with a $W$ boson, JHEP 07 (2008) 029 [arXiv: 0805.3067] [INSPIRE]. 
[60] H.B. Hartanto, B. Jager, L. Reina and D. Wackeroth, Higgs boson production in association with top quarks in the POWHEG BOX, Phys. Rev. D 91 (2015) 094003 [arXiv: 1501.04498] [INSPIRE].

[61] N. Kidonakis, Differential and total cross sections for top pair and single top production, in the proceedings of the $20^{\text {th }}$ International Workshop on Deep-Inelastic Scattering and Related Subjects, March 26-30, Bonn, Germany (2012), arXiv:1205.3453 [INSPIRE].

[62] M. Cacciari et al., Top-pair production at hadron colliders with next-to-next-to-leading logarithmic soft-gluon resummation, Phys. Lett. B $\mathbf{7 1 0}$ (2012) 612 [arXiv:1111.5869] [INSPIRE].

[63] P. Bärnreuther, M. Czakon and A. Mitov, Percent level precision physics at the Tevatron: first genuine NNLO QCD corrections to $q \bar{q} \rightarrow t \bar{t}+X$, Phys. Rev. Lett. 109 (2012) 132001 [arXiv: 1204.5201] [INSPIRE].

[64] M. Czakon and A. Mitov, NNLO corrections to top-pair production at hadron colliders: the all-fermionic scattering channels, JHEP 12 (2012) 054 [arXiv:1207.0236] [INSPIRE].

[65] M. Czakon and A. Mitov, NNLO corrections to top pair production at hadron colliders: the quark-gluon reaction, JHEP 01 (2013) 080 [arXiv:1210.6832] [INSPIRE].

[66] M. Beneke, P. Falgari, S. Klein and C. Schwinn, Hadronic top-quark pair production with NNLL threshold resummation, Nucl. Phys. B 855 (2012) 695 [arXiv:1109.1536] [INSPIRE].

[67] M. Czakon, P. Fiedler and A. Mitov, Total top-quark pair-production cross section at hadron colliders through $O\left(\alpha_{S}^{4}\right)$, Phys. Rev. Lett. 110 (2013) 252004 [arXiv:1303.6254] [InSPIRE].

[68] M. Czakon and A. Mitov, Top++: a program for the calculation of the top-pair cross-section at hadron colliders, Comput. Phys. Commun. 185 (2014) 2930 [arXiv:1112.5675] [INSPIRE].

[69] M. Aliev et al., HATHOR: HAdronic Top and Heavy quarks crOss section calculatoR, Comput. Phys. Commun. 182 (2011) 1034 [arXiv:1007.1327] [InSPIRE].

[70] P. Kant et al., HatHor for single top-quark production: Updated predictions and uncertainty estimates for single top-quark production in hadronic collisions, Comput. Phys. Commun. 191 (2015) 74 [arXiv:1406.4403] [INSPIRE].

[71] F. Maltoni, D. Pagani and I. Tsinikos, Associated production of a top-quark pair with vector bosons at NLO in QCD: impact on $t \bar{t} H$ searches at the LHC, JHEP 02 (2016) 113 [arXiv: 1507.05640] [INSPIRE].

[72] J.M. Campbell, R. Ellis and C. Williams, Vector boson pair production at the LHC, JHEP 07 (2011) 018 [arXiv: 1105.0020] [INSPIRE].

[73] N. Kidonakis, Two-loop soft anomalous dimensions for single top quark associated production with a $W^{-}$or $H^{-}$, Phys. Rev. D 82 (2010) 054018 [arXiv: 1005.4451] [INSPIRE].

[74] NNPDF collaboration, Unbiased global determination of parton distributions and their uncertainties at NNLO and at LO, Nucl. Phys. B 855 (2012) 153 [arXiv:1107.2652] [INSPIRE].

[75] T. Sjöstrand et al., An introduction to PYTHIA 8.2, Comput. Phys. Commun. 191 (2015) 159 [arXiv:1410.3012] [INSPIRE].

[76] CMS collaboration, Study of the underlying event in top quark pair production in pp collisions at $13 \mathrm{TeV}$, Eur. Phys. J. C 79 (2019) 123 [arXiv: 1807.02810] [InSPIRE]. 
[77] P. Skands, S. Carrazza and J. Rojo, Tuning PYTHIA 8.1: the Monash 2013 Tune, Eur. Phys. J. C 74 (2014) 3024 [arXiv: 1404.5630] [InSPIRE].

[78] CMS collaboration, Event generator tunes obtained from underlying event and multiparton scattering measurements, Eur. Phys. J. C 76 (2016) 155 [arXiv:1512.00815] [INSPIRE].

[79] GEANT4 collaboration, GEANT4 - A simulation toolkit, Nucl. Instrum. Meth. A 506 (2003) 250 [INSPIRE].

[80] CMS collaboration, Measurement of the inelastic proton-proton cross section at $\sqrt{s}=13 \mathrm{TeV}$, JHEP 07 (2018) 161 [arXiv:1802.02613] [INSPIRE].

[81] CMS collaboration, Particle-flow reconstruction and global event description with the CMS detector, 2017 JINST 12 P10003 [arXiv:1706. 04965] [INSPIRE].

[82] CMS collaboration, Performance of electron reconstruction and selection with the CMS detector in proton-proton collisions at $\sqrt{s}=8 \mathrm{TeV}, 2015$ JINST $10 \mathrm{P} 06005$ [arXiv: 1502.02701] [INSPIRE].

[83] CMS collaboration, Performance of the CMS muon detector and muon reconstruction with proton-proton collisions at $\sqrt{s}=13 \mathrm{TeV}, 2018$ JINST $13 \mathrm{P} 06015$ [arXiv: 1804.04528] [INSPIRE].

[84] CMS collaboration, Performance of reconstruction and identification of $\tau$ leptons decaying to hadrons and $\nu_{\tau}$ in pp collisions at $\sqrt{s}=13 \mathrm{TeV}, 2018$ JINST $13 \mathrm{P} 10005$ [arXiv: 1809.02816] [INSPIRE].

[85] CMS collaboration, Reconstruction and identification of $\tau$ lepton decays to hadrons and $\nu_{\tau}$ at CMS, 2016 JINST 11 P01019 [arXiv:1510.07488] [INSPIRE].

[86] M. Cacciari, G.P. Salam and G. Soyez, The anti- $k_{t}$ jet clustering algorithm, JHEP 04 (2008) 063 [arXiv: 0802.1189] [INSPIRE].

[87] M. Cacciari, G.P. Salam and G. Soyez, FastJet user manual, Eur. Phys. J. C 72 (2012) 1896 [arXiv:1111.6097] [INSPIRE].

[88] CMS collaboration, Pileup mitigation at CMS in $13 \mathrm{TeV}$ data, CMS-PAS-JME-18-001 (2019).

[89] CMS collaboration, Jet energy scale and resolution in the CMS experiment in pp collisions at $8 \mathrm{TeV}, 2017$ JINST $12 \mathrm{P} 02014$ [arXiv:1607.03663] [INSPIRE].

[90] CMS collaboration, Identification of heavy-flavour jets with the CMS detector in pp collisions at $13 \mathrm{TeV}, 2018$ JINST 13 P05011 [arXiv: 1712.07158] [INSPIRE].

[91] CMS collaboration, Performance of missing transverse momentum reconstruction in proton-proton collisions at $\sqrt{s}=13$ TeV using the CMS detector, 2019 JINST 14 P07004 [arXiv: 1903.06078] [INSPIRE].

[92] D. Krohn, J. Thaler and L.-T. Wang, Jet trimming, JHEP 02 (2010) 084 [arXiv: 0912.1342] [INSPIRE].

[93] CMS collaboration, Jet algorithms performance in $13 \mathrm{TeV}$ data, CMS-PAS-JME-16-003 (2017).

[94] A.J. Larkoski, S. Marzani, G. Soyez and J. Thaler, Soft drop, JHEP 05 (2014) 146 [arXiv: 1402.2657] [INSPIRE].

[95] CMS collaboration, Performance of quark/gluon discrimination in $8 \mathrm{TeV}$ pp data, CMS-PAS-JME-13-002 (2013).

[96] CMS collaboration, Identification of c-quark jets at the CMS experiment, CMS-PAS-BTV-16-001 (2016). 
[97] J. Thaler and K. Van Tilburg, Identifying boosted objects with $N$-subjettiness, JHEP 03 (2011) 015 [arXiv: 1011.2268] [INSPIRE].

[98] A. Hocker et al., TMVA - Toolkit for Multivariate Data Analysis, in the proceedings of the XIth International Workshop on Advanced Computing and Analysis Techniques in Physics Research (ACAT) 200\%, April 23-27, Amsterdam, Netherlands (2007), physics/0703039 [INSPIRE].

[99] CMS collaboration, Search for direct production of supersymmetric partners of the top quark in the all-jets final state in proton-proton collisions at $\sqrt{s}=13$ TeV, JHEP 10 (2017) 005 [arXiv: 1707.03316] [INSPIRE].

[100] ATLAS collaboration, Measurement of the inelastic proton-proton cross section at $\sqrt{s}=13 \mathrm{TeV}$ with the ATLAS detector at the LHC, Phys. Rev. Lett. 117 (2016) 182002 [arXiv: 1606. 02625] [INSPIRE].

[101] CMS collaboration, CMS luminosity measurements for the 2016 data taking period, CMS-PAS-LUM-17-001 (2017).

[102] J. Butterworth et al., PDF4LHC recommendations for LHC Run II, J. Phys. G 43 (2016) 023001 [arXiv: 1510.03865] [INSPIRE].

[103] R.J. Barlow and C. Beeston, Fitting using finite Monte Carlo samples, Comput. Phys. Commun. 77 (1993) 219 [INSPIRE].

[104] J.S. Conway, Incorporating nuisance parameters in likelihoods for multisource spectra, in the proceedings of the PHYSTAT 2011 Workshop on Statistical Issues Related to Discovery Claims in Search Experiments and Unfolding, January 17-20, CERN, Geneva, Switzerland (2011), arXiv:1103.0354 [INSPIRE].

[105] T. Junk, Confidence level computation for combining searches with small statistics, Nucl. Instrum. Meth. A 434 (1999) 435 [hep-ex/9902006] [INSPIRE].

[106] A.L. Read, Presentation of search results: the CL(s) technique, [INSPIRE].

[107] G. Cowan, K. Cranmer, E. Gross and O. Vitells, Asymptotic formulae for likelihood-based tests of new physics, Eur. Phys. J. C 71 (2011) 1554 [Erratum ibid. 73 (2013) 2501] [arXiv: 1007.1727] [INSPIRE].

[108] ATLAS and CMS collaborations, Procedure for the LHC Higgs boson search combination in Summer 2011, CMS-NOTE-2011-005 (2011).

[109] A. Djouadi and J. Quevillon, The MSSM Higgs sector at a high $M_{\mathrm{SUSY}}$ : reopening the low $\tan \beta$ regime and heavy Higgs searches, JHEP 10 (2013) 028 [arXiv:1304.1787] [INSPIRE].

[110] L. Maiani, A.D. Polosa and V. Riquer, Bounds to the Higgs sector masses in minimal supersymmetry from LHC data, Phys. Lett. B $\mathbf{7 2 4}$ (2013) 274 [arXiv:1305.2172] [INSPIRE].

[111] A. Djouadi et al., The post-Higgs MSSM scenario: habemus MSSM?, Eur. Phys. J. C 73 (2013) 2650 [arXiv: 1307.5205] [INSPIRE].

[112] A. Djouadi et al., Fully covering the MSSM Higgs sector at the LHC, JHEP 06 (2015) 168 [arXiv: 1502.05653] [INSPIRE].

[113] E. Bagnaschi et al., MSSM Higgs boson searches at the LHC: benchmark scenarios for Run 2 and beyond, Eur. Phys. J. C $\mathbf{7 9}$ (2019) 617 [arXiv:1808.07542] [INSPIRE].

[114] S. Heinemeyer, W. Hollik and G. Weiglein, The masses of the neutral CP-even Higgs bosons in the MSSM: accurate analysis at the two loop level, Eur. Phys. J. C 9 (1999) 343 [hep-ph/9812472] [INSPIRE]. 
[115] G. Degrassi et al., Towards high precision predictions for the MSSM Higgs sector, Eur. Phys. J. C 28 (2003) 133 [hep-ph/0212020] [INSPIRE].

[116] M. Frank et al., The Higgs boson masses and mixings of the complex MSSM in the Feynman-diagrammatic approach, JHEP 02 (2007) 047 [hep-ph/0611326] [INSPIRE].

[117] T. Hahn et al., High-precision predictions for the light CP-even Higgs boson mass of the minimal supersymmetric standard model, Phys. Rev. Lett. 112 (2014) 141801 [arXiv:1312.4937] [INSPIRE].

[118] H. Bahl and W. Hollik, Precise prediction for the light MSSM Higgs boson mass combining effective field theory and fixed-order calculations, Eur. Phys. J. C 76 (2016) 499 [arXiv: 1608.01880] [INSPIRE].

[119] H. Bahl, S. Heinemeyer, W. Hollik and G. Weiglein, Reconciling EFT and hybrid calculations of the light MSSM Higgs-boson mass, Eur. Phys. J. C 78 (2018) 57 [arXiv: 1706. 00346] [INSPIRE].

[120] A. Djouadi, M.M. Muhlleitner and M. Spira, Decays of supersymmetric particles: The Program SUSY-HIT (SUspect-SdecaY-HDECAY-InTerface), Acta Phys. Polon. B 38 (2007) 635 [hep-ph/0609292] [INSPIRE].

[121] A. Djouadi, J. Kalinowski, M. Muehlleitner and M. Spira, HDECAY: Twenty ${ }_{++}$years after, Comput. Phys. Commun. 238 (2019) 214 [arXiv:1801.09506] [InSPIRE].

[122] A. Bredenstein, A. Denner, S. Dittmaier and M.M. Weber, Precise predictions for the Higgs-boson decay $H \rightarrow W W / Z Z \rightarrow 4$ leptons, Phys. Rev. D 74 (2006) 013004 [hep-ph/0604011] [INSPIRE].

[123] A. Bredenstein, A. Denner, S. Dittmaier and M.M. Weber, Radiative corrections to the semileptonic and hadronic Higgs-boson decays $H \rightarrow W W / Z Z \rightarrow 4$ fermions, JHEP 02 (2007) 080 [hep-ph/0611234] [INSPIRE]. 


\section{The CMS collaboration}

\section{Yerevan Physics Institute, Yerevan, Armenia}

A.M. Sirunyan ${ }^{\dagger}$, A. Tumasyan

\section{Institut für Hochenergiephysik, Wien, Austria}

W. Adam, F. Ambrogi, T. Bergauer, J. Brandstetter, M. Dragicevic, J. Erö, A. Escalante Del Valle, M. Flechl, R. Frühwirth ${ }^{1}$, M. Jeitler ${ }^{1}$, N. Krammer, I. Krätschmer, D. Liko, T. Madlener, I. Mikulec, N. Rad, J. Schieck ${ }^{1}$, R. Schöfbeck, M. Spanring, D. Spitzbart, W. Waltenberger, C.-E. Wulz ${ }^{1}$, M. Zarucki

Institute for Nuclear Problems, Minsk, Belarus

V. Drugakov, V. Mossolov, J. Suarez Gonzalez

Universiteit Antwerpen, Antwerpen, Belgium

M.R. Darwish, E.A. De Wolf, D. Di Croce, X. Janssen, A. Lelek, M. Pieters, H. Rejeb Sfar, H. Van Haevermaet, P. Van Mechelen, S. Van Putte, N. Van Remortel

\section{Vrije Universiteit Brussel, Brussel, Belgium}

F. Blekman, E.S. Bols, S.S. Chhibra, J. D'Hondt, J. De Clercq, D. Lontkovskyi, S. Lowette, I. Marchesini, S. Moortgat, Q. Python, K. Skovpen, S. Tavernier, W. Van Doninck, P. Van Mulders

\section{Université Libre de Bruxelles, Bruxelles, Belgium}

D. Beghin, B. Bilin, H. Brun, B. Clerbaux, G. De Lentdecker, H. Delannoy, B. Dorney, L. Favart, A. Grebenyuk, A.K. Kalsi, A. Popov, N. Postiau, E. Starling, L. Thomas, C. Vander Velde, P. Vanlaer, D. Vannerom

\section{Ghent University, Ghent, Belgium}

T. Cornelis, D. Dobur, I. Khvastunov ${ }^{2}$, M. Niedziela, C. Roskas, M. Tytgat, W. Verbeke, B. Vermassen, M. Vit

\section{Université Catholique de Louvain, Louvain-la-Neuve, Belgium}

O. Bondu, G. Bruno, C. Caputo, P. David, C. Delaere, M. Delcourt, A. Giammanco, V. Lemaitre, J. Prisciandaro, A. Saggio, M. Vidal Marono, P. Vischia, J. Zobec

Centro Brasileiro de Pesquisas Fisicas, Rio de Janeiro, Brazil

F.L. Alves, G.A. Alves, G. Correia Silva, C. Hensel, A. Moraes, P. Rebello Teles

Universidade do Estado do Rio de Janeiro, Rio de Janeiro, Brazil

E. Belchior Batista Das Chagas, W. Carvalho, J. Chinellato ${ }^{3}$, E. Coelho, E.M. Da Costa, G.G. Da Silveira ${ }^{4}$, D. De Jesus Damiao, C. De Oliveira Martins, S. Fonseca De Souza, L.M. Huertas Guativa, H. Malbouisson, J. Martins ${ }^{5}$, D. Matos Figueiredo, M. Medina Jaime ${ }^{6}$, M. Melo De Almeida, C. Mora Herrera, L. Mundim, H. Nogima, W.L. Prado Da Silva, L.J. Sanchez Rosas, A. Santoro, A. Sznajder, M. Thiel, E.J. Tonelli Manganote ${ }^{3}$, F. Torres Da Silva De Araujo, A. Vilela Pereira 
Universidade Estadual Paulista ${ }^{a}$, Universidade Federal do $\mathrm{ABC}^{b}$, São Paulo, Brazil

C.A. Bernardes ${ }^{a}$, L. Calligaris ${ }^{a}$, T.R. Fernandez Perez Tomei ${ }^{a}$, E.M. Gregores ${ }^{b}$, D.S. Lemos, P.G. Mercadante ${ }^{b}$, S.F. Novaes ${ }^{a}$, SandraS. Padula ${ }^{a}$

Institute for Nuclear Research and Nuclear Energy, Bulgarian Academy of Sciences, Sofia, Bulgaria

A. Aleksandrov, G. Antchev, R. Hadjiiska, P. Iaydjiev, M. Misheva, M. Rodozov, M. Shopova, G. Sultanov

University of Sofia, Sofia, Bulgaria

M. Bonchev, A. Dimitrov, T. Ivanov, L. Litov, B. Pavlov, P. Petkov

Beihang University, Beijing, China

W. Fang ${ }^{7}$, X. Gao ${ }^{7}$, L. Yuan

Department of Physics, Tsinghua University, Beijing, China

M. Ahmad, Z. Hu, Y. Wang

Institute of High Energy Physics, Beijing, China

G.M. Chen, H.S. Chen, M. Chen, C.H. Jiang, D. Leggat, H. Liao, Z. Liu, A. Spiezia, J. Tao, E. Yazgan, H. Zhang, S. Zhang ${ }^{8}$, J. Zhao

State Key Laboratory of Nuclear Physics and Technology, Peking University, Beijing, China

A. Agapitos, Y. Ban, G. Chen, A. Levin, J. Li, L. Li, Q. Li, Y. Mao, S.J. Qian, D. Wang, Q. Wang

Zhejiang University, Hangzhou, China

M. Xiao

Universidad de Los Andes, Bogota, Colombia

C. Avila, A. Cabrera, C. Florez, C.F. González Hernández, M.A. Segura Delgado

Universidad de Antioquia, Medellin, Colombia

J. Mejia Guisao, J.D. Ruiz Alvarez, C.A. Salazar González, N. Vanegas Arbelaez

University of Split, Faculty of Electrical Engineering, Mechanical Engineering and Naval Architecture, Split, Croatia

D. Giljanović, N. Godinovic, D. Lelas, I. Puljak, T. Sculac

University of Split, Faculty of Science, Split, Croatia

Z. Antunovic, M. Kovac

Institute Rudjer Boskovic, Zagreb, Croatia

V. Brigljevic, D. Ferencek, K. Kadija, B. Mesic, M. Roguljic, A. Starodumov ${ }^{9}$, T. Susa

University of Cyprus, Nicosia, Cyprus

M.W. Ather, A. Attikis, E. Erodotou, A. Ioannou, M. Kolosova, S. Konstantinou,

G. Mavromanolakis, J. Mousa, C. Nicolaou, F. Ptochos, P.A. Razis, H. Rykaczewski,

D. Tsiakkouri 
Charles University, Prague, Czech Republic

M. Finger ${ }^{10}$, M. Finger Jr. ${ }^{10}$, A. Kveton, J. Tomsa

Escuela Politecnica Nacional, Quito, Ecuador

E. Ayala

Universidad San Francisco de Quito, Quito, Ecuador

E. Carrera Jarrin

Academy of Scientific Research and Technology of the Arab Republic of Egypt, Egyptian Network of High Energy Physics, Cairo, Egypt

H. Abdalla ${ }^{11}$, S. Elgammal ${ }^{12}$

National Institute of Chemical Physics and Biophysics, Tallinn, Estonia

S. Bhowmik, A. Carvalho Antunes De Oliveira, R.K. Dewanjee, K. Ehataht, M. Kadastik, M. Raidal, C. Veelken

Department of Physics, University of Helsinki, Helsinki, Finland

P. Eerola, L. Forthomme, H. Kirschenmann, K. Osterberg, M. Voutilainen

Helsinki Institute of Physics, Helsinki, Finland

F. Garcia, J. Havukainen, J.K. Heikkilä, V. Karimäki, M.S. Kim, R. Kinnunen, T. Lampén,

K. Lassila-Perini, S. Laurila, S. Lehti, T. Lindén, P. Luukka, T. Mäenpää, H. Siikonen,

E. Tuominen, J. Tuominiemi

Lappeenranta University of Technology, Lappeenranta, Finland

T. Tuuva

IRFU, CEA, Université Paris-Saclay, Gif-sur-Yvette, France

M. Besancon, F. Couderc, M. Dejardin, D. Denegri, B. Fabbro, J.L. Faure, F. Ferri,

S. Ganjour, A. Givernaud, P. Gras, G. Hamel de Monchenault, P. Jarry, C. Leloup,

B. Lenzi, E. Locci, J. Malcles, J. Rander, A. Rosowsky, M.Ö. Sahin, A. Savoy-Navarro ${ }^{13}$,

M. Titov

Laboratoire Leprince-Ringuet, CNRS/IN2P3, Ecole Polytechnique, Institut Polytechnique de Paris

S. Ahuja, C. Amendola, F. Beaudette, P. Busson, C. Charlot, B. Diab, G. Falmagne,

R. Granier de Cassagnac, I. Kucher, A. Lobanov, C. Martin Perez, M. Nguyen, C. Ochando,

P. Paganini, J. Rembser, R. Salerno, J.B. Sauvan, Y. Sirois, A. Zabi, A. Zghiche

Université de Strasbourg, CNRS, IPHC UMR 7178, Strasbourg, France

J.-L. Agram ${ }^{14}$, J. Andrea, D. Bloch, G. Bourgatte, J.-M. Brom, E.C. Chabert, C. Collard,

E. Conte ${ }^{14}$, J.-C. Fontaine ${ }^{14}$, D. Gelé, U. Goerlach, M. Jansová, A.-C. Le Bihan, N. Tonon,

P. Van Hove

Centre de Calcul de l'Institut National de Physique Nucleaire et de Physique des Particules, CNRS/IN2P3, Villeurbanne, France

S. Gadrat 
Université de Lyon, Université Claude Bernard Lyon 1, CNRS-IN2P3, Institut de Physique Nucléaire de Lyon, Villeurbanne, France

S. Beauceron, C. Bernet, G. Boudoul, C. Camen, A. Carle, N. Chanon, R. Chierici, D. Contardo, P. Depasse, H. El Mamouni, J. Fay, S. Gascon, M. Gouzevitch, B. Ille, Sa. Jain, F. Lagarde, I.B. Laktineh, H. Lattaud, A. Lesauvage, M. Lethuillier, L. Mirabito, S. Perries, V. Sordini, L. Torterotot, G. Touquet, M. Vander Donckt, S. Viret

Georgian Technical University, Tbilisi, Georgia

A. Khvedelidze ${ }^{10}$

Tbilisi State University, Tbilisi, Georgia

Z. Tsamalaidze ${ }^{10}$

RWTH Aachen University, I. Physikalisches Institut, Aachen, Germany

C. Autermann, L. Feld, M.K. Kiesel, K. Klein, M. Lipinski, D. Meuser, A. Pauls, M. Preuten, M.P. Rauch, J. Schulz, M. Teroerde, B. Wittmer

RWTH Aachen University, III. Physikalisches Institut A, Aachen, Germany

M. Erdmann, B. Fischer, S. Ghosh, T. Hebbeker, K. Hoepfner, H. Keller, L. Mastrolorenzo, M. Merschmeyer, A. Meyer, P. Millet, G. Mocellin, S. Mondal, S. Mukherjee, D. Noll, A. Novak, T. Pook, A. Pozdnyakov, T. Quast, M. Radziej, Y. Rath, H. Reithler, J. Roemer, A. Schmidt, S.C. Schuler, A. Sharma, S. Wiedenbeck, S. Zaleski

RWTH Aachen University, III. Physikalisches Institut B, Aachen, Germany G. Flügge, W. Haj Ahmad ${ }^{15}$, O. Hlushchenko, T. Kress, T. Müller, A. Nowack, C. Pistone, O. Pooth, D. Roy, H. Sert, A. Stahl ${ }^{16}$

\section{Deutsches Elektronen-Synchrotron, Hamburg, Germany}

M. Aldaya Martin, P. Asmuss, I. Babounikau, H. Bakhshiansohi, K. Beernaert, O. Behnke, A. Bermúdez Martínez, D. Bertsche, A.A. Bin Anuar, K. Borras ${ }^{17}$, V. Botta, A. Campbell, A. Cardini, P. Connor, S. Consuegra Rodríguez, C. Contreras-Campana, V. Danilov, A. De Wit, M.M. Defranchis, C. Diez Pardos, D. Domínguez Damiani, G. Eckerlin, D. Eckstein, T. Eichhorn, A. Elwood, E. Eren, E. Gallo ${ }^{18}$, A. Geiser, A. Grohsjean, M. Guthoff, M. Haranko, A. Harb, A. Jafari, N.Z. Jomhari, H. Jung, A. Kasem ${ }^{17}$, M. Kasemann, H. Kaveh, J. Keaveney, C. Kleinwort, J. Knolle, D. Krücker, W. Lange, T. Lenz, J. Lidrych, K. Lipka, W. Lohmann ${ }^{19}$, R. Mankel, I.-A. Melzer-Pellmann, A.B. Meyer, M. Meyer, M. Missiroli, G. Mittag, J. Mnich, A. Mussgiller, V. Myronenko, D. Pérez Adán, S.K. Pflitsch, D. Pitzl, A. Raspereza, A. Saibel, M. Savitskyi, V. Scheurer, P. Schütze, C. Schwanenberger, R. Shevchenko, A. Singh, H. Tholen, O. Turkot, A. Vagnerini, M. Van De Klundert, R. Walsh, Y. Wen, K. Wichmann, C. Wissing, O. Zenaiev, R. Zlebcik

\section{University of Hamburg, Hamburg, Germany}

R. Aggleton, S. Bein, L. Benato, A. Benecke, V. Blobel, T. Dreyer, A. Ebrahimi, F. Feindt, A. Fröhlich, C. Garbers, E. Garutti, D. Gonzalez, P. Gunnellini, J. Haller, A. Hinzmann, A. Karavdina, G. Kasieczka, R. Klanner, R. Kogler, N. Kovalchuk, S. Kurz, V. Kutzner, J. Lange, T. Lange, A. Malara, J. Multhaup, C.E.N. Niemeyer, A. Perieanu, A. Reimers, 
O. Rieger, C. Scharf, P. Schleper, S. Schumann, J. Schwandt, J. Sonneveld, H. Stadie, G. Steinbrück, F.M. Stober, B. Vormwald, I. Zoi

Karlsruher Institut fuer Technologie, Karlsruhe, Germany

M. Akbiyik, C. Barth, M. Baselga, S. Baur, T. Berger, E. Butz, R. Caspart, T. Chwalek, W. De Boer, A. Dierlamm, K. El Morabit, N. Faltermann, M. Giffels, P. Goldenzweig, A. Gottmann, M.A. Harrendorf, F. Hartmann ${ }^{16}$, U. Husemann, S. Kudella, S. Mitra, M.U. Mozer, D. Müller, Th. Müller, M. Musich, A. Nürnberg, G. Quast, K. Rabbertz, M. Schröder, I. Shvetsov, H.J. Simonis, R. Ulrich, M. Wassmer, M. Weber, C. Wöhrmann, R. Wolf

Institute of Nuclear and Particle Physics (INPP), NCSR Demokritos, Aghia Paraskevi, Greece

G. Anagnostou, P. Asenov, G. Daskalakis, T. Geralis, A. Kyriakis, D. Loukas, G. Paspalaki National and Kapodistrian University of Athens, Athens, Greece

M. Diamantopoulou, G. Karathanasis, P. Kontaxakis, A. Manousakis-katsikakis, A. Panagiotou, I. Papavergou, N. Saoulidou, A. Stakia, K. Theofilatos, K. Vellidis, E. Vourliotis

National Technical University of Athens, Athens, Greece

G. Bakas, K. Kousouris, I. Papakrivopoulos, G. Tsipolitis

University of Ioánnina, Ioánnina, Greece

I. Evangelou, C. Foudas, P. Gianneios, P. Katsoulis, P. Kokkas, S. Mallios, K. Manitara, N. Manthos, I. Papadopoulos, J. Strologas, F.A. Triantis, D. Tsitsonis

MTA-ELTE Lendület CMS Particle and Nuclear Physics Group, Eötvös Loránd University, Budapest, Hungary

M. Bartók ${ }^{20}$, R. Chudasama, M. Csanad, P. Major, K. Mandal, A. Mehta, M.I. Nagy, G. Pasztor, O. Surányi, G.I. Veres

Wigner Research Centre for Physics, Budapest, Hungary

G. Bencze, C. Hajdu, D. Horvath ${ }^{21}$, F. Sikler, T.Á. Vámi, V. Veszpremi, G. Vesztergombi ${ }^{\dagger}$

Institute of Nuclear Research ATOMKI, Debrecen, Hungary

N. Beni, S. Czellar, J. Karancsi ${ }^{20}$, A. Makovec, J. Molnar, Z. Szillasi

Institute of Physics, University of Debrecen, Debrecen, Hungary

P. Raics, D. Teyssier, Z.L. Trocsanyi, B. Ujvari

Eszterhazy Karoly University, Karoly Robert Campus, Gyongyos, Hungary

T. Csorgo, W.J. Metzger, F. Nemes, T. Novak

Indian Institute of Science (IISc), Bangalore, India

S. Choudhury, J.R. Komaragiri, P.C. Tiwari

National Institute of Science Education and Research, HBNI, Bhubaneswar, India

S. Bahinipati ${ }^{23}$, C. Kar, G. Kole, P. Mal, V.K. Muraleedharan Nair Bindhu, A. Nayak ${ }^{24}$, D.K. Sahoo ${ }^{23}$, S.K. Swain 
Panjab University, Chandigarh, India

S. Bansal, S.B. Beri, V. Bhatnagar, S. Chauhan, R. Chawla, N. Dhingra, R. Gupta, A. Kaur, M. Kaur, S. Kaur, P. Kumari, M. Lohan, M. Meena, K. Sandeep, S. Sharma, J.B. Singh, A.K. Virdi

University of Delhi, Delhi, India

A. Bhardwaj, B.C. Choudhary, R.B. Garg, M. Gola, S. Keshri, Ashok Kumar, M. Naimuddin, P. Priyanka, K. Ranjan, Aashaq Shah, R. Sharma

Saha Institute of Nuclear Physics, HBNI, Kolkata, India

R. Bhardwaj ${ }^{25}$, M. Bharti ${ }^{25}$, R. Bhattacharya, S. Bhattacharya, U. Bhawandeep ${ }^{25}$,

D. Bhowmik, S. Dutta, S. Ghosh, B. Gomber ${ }^{26}$, M. Maity ${ }^{27}$, K. Mondal, S. Nandan, A. Purohit, P.K. Rout, G. Saha, S. Sarkar, T. Sarkar ${ }^{27}$, M. Sharan, B. Singh ${ }^{25}$, S. Thakur ${ }^{25}$

Indian Institute of Technology Madras, Madras, India

P.K. Behera, P. Kalbhor, A. Muhammad, P.R. Pujahari, A. Sharma, A.K. Sikdar

Bhabha Atomic Research Centre, Mumbai, India

D. Dutta, V. Jha, V. Kumar, D.K. Mishra, P.K. Netrakanti, L.M. Pant, P. Shukla

Tata Institute of Fundamental Research-A, Mumbai, India

T. Aziz, M.A. Bhat, S. Dugad, G.B. Mohanty, N. Sur, RavindraKumar Verma

Tata Institute of Fundamental Research-B, Mumbai, India

S. Banerjee, S. Bhattacharya, S. Chatterjee, P. Das, M. Guchait, S. Karmakar, S. Kumar, G. Majumder, K. Mazumdar, N. Sahoo, S. Sawant

Indian Institute of Science Education and Research (IISER), Pune, India

S. Dube, V. Hegde, B. Kansal, A. Kapoor, K. Kothekar, S. Pandey, A. Rane, A. Rastogi, S. Sharma

Institute for Research in Fundamental Sciences (IPM), Tehran, Iran

S. Chenarani ${ }^{28}$, E. Eskandari Tadavani, S.M. Etesami ${ }^{28}$, M. Khakzad, M. Mohammadi Najafabadi, M. Naseri, F. Rezaei Hosseinabadi

University College Dublin, Dublin, Ireland

M. Felcini, M. Grunewald

INFN Sezione di Bari ${ }^{a}$, Università di Bari ${ }^{b}$, Politecnico di Bari ${ }^{c}$, Bari, Italy

M. Abbrescia ${ }^{a, b}$, R. Aly ${ }^{a, b, 29}$, C. Calabria ${ }^{a, b}$, A. Colaleo ${ }^{a}$, D. Creanza ${ }^{a, c}$, L. Cristella $^{a, b}$, N. De Filippis ${ }^{a, c}$, M. De Palma ${ }^{a, b}$, A. Di Florio ${ }^{a, b}$, W. Elmetenawee ${ }^{a, b}$ L. Fiore $^{a}$, A. Gelmi ${ }^{a, b}$, G. Iaselli ${ }^{a, c}$, M. Ince ${ }^{a, b}, \mathrm{~S}_{\text {Lezki }}{ }^{a, b}$, G. Maggi $^{a, c}$, M. Maggi $^{a}$, G. Miniello $^{a, b}$, S. $\mathrm{My}^{a, b}$, S. Nuzzo ${ }^{a, b}$, A. Pompili ${ }^{a, b}$, G. Pugliese ${ }^{a, c}$, R. Radogna ${ }^{a}$, A. Ranieri ${ }^{a}$, G. Selvaggi ${ }^{a, b}$, L. Silvestris ${ }^{a}$, F.M. Simone ${ }^{a, b}$, R. Venditti ${ }^{a}$, P. Verwilligen ${ }^{a}$

INFN Sezione di Bologna ${ }^{a}$, Università di Bologna ${ }^{b}$, Bologna, Italy

G. Abbiendi ${ }^{a}$, C. Battilana ${ }^{a, b}$, D. Bonacorsi ${ }^{a, b}$, L. Borgonovi $^{a, b}$, S. Braibant-Giacomelli $^{a, b}$, R. Campanini ${ }^{a, b}$, P. Capiluppi ${ }^{a, b}$, A. Castro ${ }^{a, b}$, F.R. Cavallo $^{a}$, C. Ciocca $^{a}$, G. Codispoti $^{a, b}$, M. Cuffiani ${ }^{a, b}$, G.M. Dallavalle ${ }^{a}$, F. Fabbri ${ }^{a}$, A. Fanfani ${ }^{a, b}$, E. Fontanesi ${ }^{a, b}$, P. Giacomelli ${ }^{a}$, 
C. Grandi ${ }^{a}$, L. Guiducci ${ }^{a, b}$, F. Iemmi ${ }^{a, b}$, S. Lo Meo ${ }^{a, 30}$, S. Marcellini ${ }^{a}$, G. Masetti ${ }^{a}$,

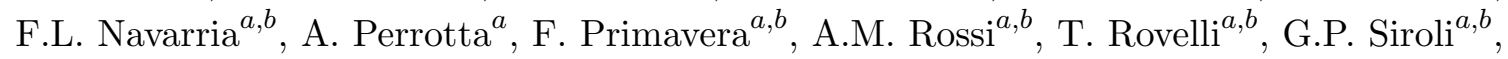
N. $\operatorname{Tosi}^{a}$

INFN Sezione di Catania $^{a}$, Università di Catania ${ }^{b}$, Catania, Italy

S. Albergo ${ }^{a, b, 31}$, S. Costa ${ }^{a, b}$, A. Di Mattia ${ }^{a}$, R. Potenza ${ }^{a, b}$, A. Tricomi ${ }^{a, b, 31}$, C. Tuve $^{a, b}$

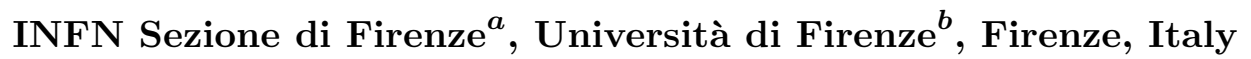

G. Barbagli ${ }^{a}$, A. Cassese, R. Ceccarelli, V. Ciulli ${ }^{a, b}$, C. Civinini ${ }^{a}$, R. D'Alessandro ${ }^{a, b}$,

E. Focardi ${ }^{a, b}$, G. Latino ${ }^{a, b}$, P. Lenzi ${ }^{a, b}$, M. Meschini ${ }^{a}$, S. Paoletti ${ }^{a}$, G. Sguazzoni ${ }^{a}$,

L. Viliani ${ }^{a}$

INFN Laboratori Nazionali di Frascati, Frascati, Italy

L. Benussi, S. Bianco, D. Piccolo

INFN Sezione di Genova ${ }^{a}$, Università di Genova ${ }^{b}$, Genova, Italy

M. Bozzo ${ }^{a, b}$, F. Ferro $^{a}$, R. Mulargia ${ }^{a, b}$, E. Robutti $^{a}$, S. Tosi $^{a, b}$

INFN Sezione di Milano-Bicocca ${ }^{a}$, Università di Milano-Bicocca ${ }^{b}$, Milano, Italy

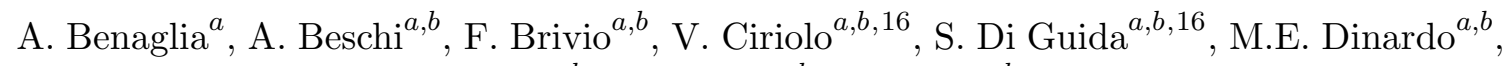
P. Dini ${ }^{a}$, S. Gennai ${ }^{a}$, A. Ghezzi ${ }^{a, b}$, P. Govoni ${ }^{a, b}$, L. Guzzi $^{a}, b$, M. Malberti ${ }^{a}$, S. Malvezzi $^{a}$, D. Menasce ${ }^{a}$, F. Monti ${ }^{a, b}$, L. Moroni $^{a}$, M. Paganoni ${ }^{a, b}$, D. Pedrini ${ }^{a}$, S. Ragazzi $^{a, b}$, T. Tabarelli de Fatis ${ }^{a, b}$, D. Zuolo ${ }^{a, b}$

INFN Sezione di Napoli ${ }^{a}$, Università di Napoli 'Federico II'b , Napoli, Italy, Università della Basilicata $^{c}$, Potenza, Italy, Università G. Marconi ${ }^{d}$, Roma, Italy

S. Buontempo ${ }^{a}$, N. Cavallo ${ }^{a, c}$, A. De Iorio ${ }^{a, b}$, A. Di Crescenzo ${ }^{a, b}$, F. Fabozzi $^{a, c}$, F. Fienga $^{a}$, G. Galati ${ }^{a}$, A.O.M. Iorio ${ }^{a, b}$, L. Lista ${ }^{a, b}$, S. Meola ${ }^{a, d, 16}$, P. Paolucci ${ }^{a, 16}$, B. Rossi ${ }^{a}$, C. Sciacca ${ }^{a, b}$, E. Voevodina ${ }^{a, b}$

INFN Sezione di Padova ${ }^{a}$, Università di Padova ${ }^{b}$, Padova, Italy, Università di Trento $^{c}$, Trento, Italy

P. $\operatorname{Azzi}^{a}$, N. Bacchetta ${ }^{a}$, D. Bisello ${ }^{a, b}$, A. Boletti ${ }^{a, b}$, A. Bragagnolo ${ }^{a, b}$, R. Carlin ${ }^{a, b}$, P. Checchia ${ }^{a}$, P. De Castro Manzano ${ }^{a}$, T. Dorigo ${ }^{a}$, U. Dosselli ${ }^{a}$, F. Gasparini ${ }^{a, b}$, U. Gasparini $^{a, b}$, A. Gozzelino ${ }^{a}$, S.Y. $\operatorname{Hoh}^{a, b}$, P. Lujan ${ }^{a}$, M. Margoni $^{a, b}$, A.T. Meneguzzo ${ }^{a, b}$, J. Pazzini ${ }^{a, b}$, M. Presilla ${ }^{b}$, P. Ronchese ${ }^{a, b}$, R. Rossin ${ }^{a, b}$, F. Simonetto ${ }^{a, b}$, A. Tiko ${ }^{a}$, M. Tosi ${ }^{a, b}$, M. Zanetti ${ }^{a, b}$, P. Zotto ${ }^{a, b}$, G. Zumerle ${ }^{a, b}$

INFN Sezione di Pavia ${ }^{a}$, Università di Pavia ${ }^{b}$, Pavia, Italy

A. Braghieri ${ }^{a}$, D. Fiorina ${ }^{a, b}$, P. Montagna ${ }^{a, b}$, S.P. Ratti ${ }^{a, b}$, V. $\operatorname{Re}^{a}$, M. Ressegotti $^{a, b}$, C. Riccardi ${ }^{a, b}$, P. Salvini ${ }^{a},{\text { I. } \operatorname{Vai}^{a}}^{a}$, P. Vitulo $^{a, b}$

INFN Sezione di Perugia ${ }^{a}$, Università di Perugia ${ }^{b}$, Perugia, Italy

M. Biasini ${ }^{a, b}$, G.M. Bilei ${ }^{a}$, D. Ciangottini ${ }^{a, b}$, L. Fanò ${ }^{a, b}$, P. Lariccia $^{a, b}$, R. Leonardi $^{a, b}$,

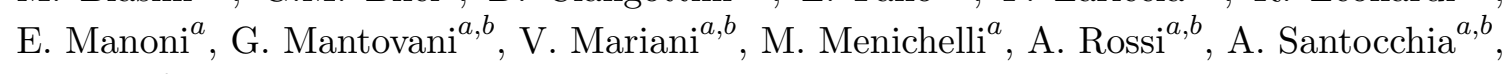

D. Spiga ${ }^{a}$ 
INFN Sezione di Pisa ${ }^{a}$, Università di Pisa ${ }^{b}$, Scuola Normale Superiore di Pisa ${ }^{c}$, Pisa, Italy

K. Androsov $^{a}$, P. Azzurri ${ }^{a}$, G. Bagliesi ${ }^{a}$, V. Bertacchi ${ }^{a, c}$, L. Bianchini $^{a}$, T. Boccali ${ }^{a}$, R. Castaldi ${ }^{a}$, M.A. Ciocci ${ }^{a}, b$, R. Dell'Orso ${ }^{a}$, S. Donato $^{a}$, G. Fedi $^{a}$, L. Giannini $^{a, c}$, A. Giassi $^{a}$, M.T. Grippo ${ }^{a}$, F. Ligabue ${ }^{a, c}$, E. Manca ${ }^{a, c}$, G. Mandorli ${ }^{a, c}$, A. Messineo ${ }^{a, b}$, F. Palla $^{a}$, A. Rizzi ${ }^{a, b}$, G. Rolandi ${ }^{32}$, S. Roy Chowdhury, A. Scribano ${ }^{a}$, P. Spagnolo $^{a}$, R. Tenchini ${ }^{a}$, G. Tonelli ${ }^{a, b}$, N. Turini, A. Venturi ${ }^{a}$, P.G. Verdini ${ }^{a}$

INFN Sezione di Roma ${ }^{a}$, Sapienza Università di Roma ${ }^{b}$, Rome, Italy

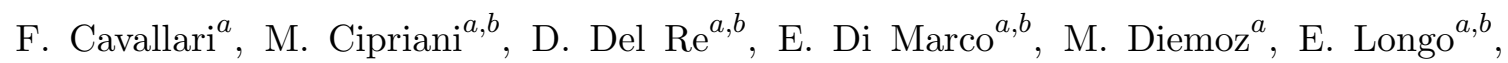
P. Meridiani ${ }^{a}$, G. Organtini ${ }^{a, b}$, F. Pandolfi ${ }^{a}$, R. Paramatti ${ }^{a, b}$, C. Quaranta ${ }^{a, b}$, S. Rahatlou ${ }^{a, b}$, C. Rovelli ${ }^{a}$, F. Santanastasio ${ }^{a, b}$, L. Soffi $^{a, b}$

INFN Sezione di Torino ${ }^{a}$, Università di Torino ${ }^{b}$, Torino, Italy, Università del Piemonte Orientale ${ }^{c}$, Novara, Italy

N. Amapane ${ }^{a, b}$, R. Arcidiacono ${ }^{a, c}$, S. Argiro ${ }^{a, b}$, M. Arneodo $^{a, c}$, N. Bartosik ${ }^{a}$, R. Bellan $^{a, b}$, A. Bellora, C. Biino ${ }^{a}$, A. Cappati ${ }^{a}, b$, N. Cartiglia ${ }^{a}$, S. Cometti $^{a},{\text { M. } \text { Costa }^{a}, b}^{a}$, R. Covarelli $^{a, b}$, N. Demaria ${ }^{a}$, B. Kiani $^{a, b}$, F. Legger, C. Mariotti ${ }^{a}$, S. Maselli $^{a}$, E. Migliore $^{a, b}$, V. Monaco $^{a, b}$, E. Monteil $^{a, b}$, M. Monteno ${ }^{a}$, M.M. Obertino ${ }^{a, b}$, G. Ortona ${ }^{a, b}$, L. Pacher $^{a, b}$, N. Pastrone ${ }^{a}$, M. Pelliccioni ${ }^{a}$, G.L. Pinna Angioni ${ }^{a, b}$, A. Romero ${ }^{a, b}$, M. Ruspa $^{a, c}$, R. Salvatico $^{a, b}$, V. Sola $^{a}$, A. Solano ${ }^{a, b}$, D. Soldi ${ }^{a, b}$, A. Staiano ${ }^{a}$, D. Trocino ${ }^{a, b}$

INFN Sezione di Trieste ${ }^{a}$, Università di Trieste ${ }^{b}$, Trieste, Italy

S. Belforte ${ }^{a}$, V. Candelise ${ }^{a, b}$, M. Casarsa ${ }^{a}$, F. Cossutti ${ }^{a}$, A. Da Rold ${ }^{a, b}$, G. Della Ricca ${ }^{a, b}$, F. Vazzoler ${ }^{a, b}$, A. Zanetti ${ }^{a}$

Kyungpook National University, Daegu, Korea

B. Kim, D.H. Kim, G.N. Kim, J. Lee, S.W. Lee, C.S. Moon, Y.D. Oh, S.I. Pak, S. Sekmen, D.C. Son, Y.C. Yang

Chonnam National University, Institute for Universe and Elementary Particles, Kwangju, Korea

H. Kim, D.H. Moon, G. Oh

Hanyang University, Seoul, Korea

B. Francois, T.J. Kim, J. Park

Korea University, Seoul, Korea

S. Cho, S. Choi, Y. Go, S. Ha, B. Hong, K. Lee, K.S. Lee, J. Lim, J. Park, S.K. Park, Y. Roh, J. Yoo

Kyung Hee University, Department of Physics

J. Goh

Sejong University, Seoul, Korea

H.S. Kim 
Seoul National University, Seoul, Korea

J. Almond, J.H. Bhyun, J. Choi, S. Jeon, J. Kim, J.S. Kim, H. Lee, K. Lee, S. Lee, K. Nam, M. Oh, S.B. Oh, B.C. Radburn-Smith, U.K. Yang, H.D. Yoo, I. Yoon, G.B. Yu

University of Seoul, Seoul, Korea

D. Jeon, H. Kim, J.H. Kim, J.S.H. Lee, I.C. Park, I.J Watson

Sungkyunkwan University, Suwon, Korea

Y. Choi, C. Hwang, Y. Jeong, J. Lee, Y. Lee, I. Yu

Riga Technical University, Riga, Latvia

V. Veckalns ${ }^{33}$

Vilnius University, Vilnius, Lithuania

V. Dudenas, A. Juodagalvis, A. Rinkevicius, G. Tamulaitis, J. Vaitkus

National Centre for Particle Physics, Universiti Malaya, Kuala Lumpur, Malaysia

Z.A. Ibrahim, F. Mohamad Idris ${ }^{34}$, W.A.T. Wan Abdullah, M.N. Yusli, Z. Zolkapli

Universidad de Sonora (UNISON), Hermosillo, Mexico

J.F. Benitez, A. Castaneda Hernandez, J.A. Murillo Quijada, L. Valencia Palomo

Centro de Investigacion y de Estudios Avanzados del IPN, Mexico City, Mexico

H. Castilla-Valdez, E. De La Cruz-Burelo, I. Heredia-De La Cruz ${ }^{35}$, R. Lopez-Fernandez,

A. Sanchez-Hernandez

Universidad Iberoamericana, Mexico City, Mexico

S. Carrillo Moreno, C. Oropeza Barrera, M. Ramirez-Garcia, F. Vazquez Valencia

Benemerita Universidad Autonoma de Puebla, Puebla, Mexico

J. Eysermans, I. Pedraza, H.A. Salazar Ibarguen, C. Uribe Estrada

Universidad Autónoma de San Luis Potosí, San Luis Potosí, Mexico

A. Morelos Pineda

University of Montenegro, Podgorica, Montenegro

J. Mijuskovic ${ }^{2}$, N. Raicevic

University of Auckland, Auckland, New Zealand

D. Krofcheck

University of Canterbury, Christchurch, New Zealand

S. Bheesette, P.H. Butler

National Centre for Physics, Quaid-I-Azam University, Islamabad, Pakistan

A. Ahmad, M. Ahmad, Q. Hassan, H.R. Hoorani, W.A. Khan, M.A. Shah, M. Shoaib, M. Waqas

AGH University of Science and Technology Faculty of Computer Science, Electronics and Telecommunications, Krakow, Poland

V. Avati, L. Grzanka, M. Malawski 
National Centre for Nuclear Research, Swierk, Poland

H. Bialkowska, M. Bluj, B. Boimska, M. Górski, M. Kazana, M. Szleper, P. Zalewski

Institute of Experimental Physics, Faculty of Physics, University of Warsaw, Warsaw, Poland

K. Bunkowski, A. Byszuk ${ }^{36}$, K. Doroba, A. Kalinowski, M. Konecki, J. Krolikowski, M. Misiura, M. Olszewski, M. Walczak

Laboratório de Instrumentação e Física Experimental de Partículas, Lisboa, Portugal

M. Araujo, P. Bargassa, D. Bastos, A. Di Francesco, P. Faccioli, B. Galinhas, M. Gallinaro,

J. Hollar, N. Leonardo, T. Niknejad, J. Seixas, K. Shchelina, G. Strong, O. Toldaiev,

J. Varela

Joint Institute for Nuclear Research, Dubna, Russia

S. Afanasiev, P. Bunin, M. Gavrilenko, I. Golutvin, I. Gorbunov, A. Kamenev, V. Karjavine, A. Lanev, A. Malakhov, V. Matveev ${ }^{37,38}$, P. Moisenz, V. Palichik, V. Perelygin, M. Savina, S. Shmatov, S. Shulha, N. Skatchkov, V. Smirnov, N. Voytishin, A. Zarubin

Petersburg Nuclear Physics Institute, Gatchina (St. Petersburg), Russia

L. Chtchipounov, V. Golovtcov, Y. Ivanov, V. Kim ${ }^{39}$, E. Kuznetsova ${ }^{40}$, P. Levchenko, V. Murzin, V. Oreshkin, I. Smirnov, D. Sosnov, V. Sulimov, L. Uvarov, A. Vorobyev

Institute for Nuclear Research, Moscow, Russia

Yu. Andreev, A. Dermenev, S. Gninenko, N. Golubev, A. Karneyeu, M. Kirsanov, N. Krasnikov, A. Pashenkov, D. Tlisov, A. Toropin

Institute for Theoretical and Experimental Physics named by A.I. Alikhanov of NRC 'Kurchatov Institute', Moscow, Russia

V. Epshteyn, V. Gavrilov, N. Lychkovskaya, A. Nikitenko ${ }^{41}$, V. Popov, I. Pozdnyakov, G. Safronov, A. Spiridonov, A. Stepennov, M. Toms, E. Vlasov, A. Zhokin

Moscow Institute of Physics and Technology, Moscow, Russia

T. Aushev

National Research Nuclear University 'Moscow Engineering Physics Institute' (MEPhI), Moscow, Russia

O. Bychkova, R. Chistov ${ }^{42}$, M. Danilov ${ }^{42}$, S. Polikarpov ${ }^{42}$, E. Tarkovskii

P.N. Lebedev Physical Institute, Moscow, Russia

V. Andreev, M. Azarkin, I. Dremin, M. Kirakosyan, A. Terkulov

Skobeltsyn Institute of Nuclear Physics, Lomonosov Moscow State University, Moscow, Russia

A. Baskakov, A. Belyaev, E. Boos, V. Bunichev, M. Dubinin ${ }^{43}$, L. Dudko, V. Klyukhin,

O. Kodolova, I. Lokhtin, S. Obraztsov, M. Perfilov, S. Petrushanko, V. Savrin

Novosibirsk State University (NSU), Novosibirsk, Russia

A. Barnyakov ${ }^{44}$, V. Blinov ${ }^{44}$, T. Dimova ${ }^{44}$, L. Kardapoltsev $^{44}$, Y. Skovpen ${ }^{44}$ 
Institute for High Energy Physics of National Research Centre 'Kurchatov Institute', Protvino, Russia

I. Azhgirey, I. Bayshev, S. Bitioukov, V. Kachanov, D. Konstantinov, P. Mandrik, V. Petrov, R. Ryutin, S. Slabospitskii, A. Sobol, S. Troshin, N. Tyurin, A. Uzunian, A. Volkov

National Research Tomsk Polytechnic University, Tomsk, Russia

A. Babaev, A. Iuzhakov, V. Okhotnikov

Tomsk State University, Tomsk, Russia

V. Borchsh, V. Ivanchenko, E. Tcherniaev

University of Belgrade: Faculty of Physics and VINCA Institute of Nuclear Sciences

P. Adzic ${ }^{45}$, P. Cirkovic, M. Dordevic, P. Milenovic, J. Milosevic, M. Stojanovic

Centro de Investigaciones Energéticas Medioambientales y Tecnológicas (CIEMAT), Madrid, Spain

M. Aguilar-Benitez, J. Alcaraz Maestre, A. Álvarez Fernández, I. Bachiller, M. Barrio Luna, CristinaF. Bedoya, J.A. Brochero Cifuentes, C.A. Carrillo Montoya, M. Cepeda, M. Cerrada, N. Colino, B. De La Cruz, A. Delgado Peris, J.P. Fernández Ramos, J. Flix, M.C. Fouz, O. Gonzalez Lopez, S. Goy Lopez, J.M. Hernandez, M.I. Josa, D. Moran, Á. Navarro Tobar, A. Pérez-Calero Yzquierdo, J. Puerta Pelayo, I. Redondo, L. Romero, S. Sánchez Navas, M.S. Soares, A. Triossi, C. Willmott

Universidad Autónoma de Madrid, Madrid, Spain

C. Albajar, J.F. de Trocóniz, R. Reyes-Almanza

Universidad de Oviedo, Instituto Universitario de Ciencias y Tecnologías Espaciales de Asturias (ICTEA), Oviedo, Spain

B. Alvarez Gonzalez, J. Cuevas, C. Erice, J. Fernandez Menendez, S. Folgueras, I. Gonzalez Caballero, J.R. González Fernández, E. Palencia Cortezon, V. Rodríguez Bouza, S. Sanchez Cruz

Instituto de Física de Cantabria (IFCA), CSIC-Universidad de Cantabria, Santander, Spain

I.J. Cabrillo, A. Calderon, B. Chazin Quero, J. Duarte Campderros, M. Fernandez, P.J. Fernández Manteca, A. García Alonso, G. Gomez, C. Martinez Rivero, P. Martinez Ruiz del Arbol, F. Matorras, J. Piedra Gomez, C. Prieels, T. Rodrigo, A. Ruiz-Jimeno, L. Russo ${ }^{46}$, L. Scodellaro, I. Vila, J.M. Vizan Garcia

University of Colombo, Colombo, Sri Lanka

K. Malagalage

University of Ruhuna, Department of Physics, Matara, Sri Lanka

W.G.D. Dharmaratna, N. Wickramage 


\section{CERN, European Organization for Nuclear Research, Geneva, Switzerland}

D. Abbaneo, B. Akgun, E. Auffray, G. Auzinger, J. Baechler, P. Baillon, A.H. Ball, D. Barney, J. Bendavid, M. Bianco, A. Bocci, P. Bortignon, E. Bossini, C. Botta, E. Brondolin, T. Camporesi, A. Caratelli, G. Cerminara, E. Chapon, G. Cucciati, D. d'Enterria, A. Dabrowski, N. Daci, V. Daponte, A. David, O. Davignon, A. De Roeck, M. Deile, M. Dobson, M. Dünser, N. Dupont, A. Elliott-Peisert, N. Emriskova, F. Fallavollita ${ }^{47}$, D. Fasanella, S. Fiorendi, G. Franzoni, J. Fulcher, W. Funk, S. Giani, D. Gigi, A. Gilbert, K. Gill, F. Glege, L. Gouskos, M. Gruchala, M. Guilbaud, D. Gulhan, J. Hegeman, C. Heidegger, Y. Iiyama, V. Innocente, T. James, P. Janot, O. Karacheban ${ }^{19}$, J. Kaspar, J. Kieseler, M. Krammer ${ }^{1}$, N. Kratochwil, C. Lange, P. Lecoq, C. Lourenço, L. Malgeri, M. Mannelli, A. Massironi, F. Meijers, J.A. Merlin, S. Mersi, E. Meschi, F. Moortgat, M. Mulders, J. Ngadiuba, J. Niedziela, S. Nourbakhsh, S. Orfanelli, L. Orsini, F. Pantaleo ${ }^{16}$, L. Pape, E. Perez, M. Peruzzi, A. Petrilli, G. Petrucciani, A. Pfeiffer, M. Pierini, F.M. Pitters, D. Rabady, A. Racz, M. Rieger, M. Rovere, H. Sakulin, C. Schäfer, C. Schwick, M. Selvaggi, A. Sharma, P. Silva, W. Snoeys, P. Sphicas ${ }^{48}$, J. Steggemann, S. Summers, V.R. Tavolaro, D. Treille, A. Tsirou, G.P. Van Onsem, A. Vartak, M. Verzetti, W.D. Zeuner

\section{Paul Scherrer Institut, Villigen, Switzerland}

L. Caminada ${ }^{49}$, K. Deiters, W. Erdmann, R. Horisberger, Q. Ingram, H.C. Kaestli, D. Kotlinski, U. Langenegger, T. Rohe, S.A. Wiederkehr

ETH Zurich - Institute for Particle Physics and Astrophysics (IPA), Zurich, Switzerland

M. Backhaus, P. Berger, N. Chernyavskaya, G. Dissertori, M. Dittmar, M. Donegà, C. Dorfer, T.A. Gómez Espinosa, C. Grab, D. Hits, W. Lustermann, R.A. Manzoni, M.T. Meinhard, F. Micheli, P. Musella, F. Nessi-Tedaldi, F. Pauss, G. Perrin, L. Perrozzi, S. Pigazzini, M.G. Ratti, M. Reichmann, C. Reissel, T. Reitenspiess, B. Ristic, D. Ruini, D.A. Sanz Becerra, M. Schönenberger, L. Shchutska, M.L. Vesterbacka Olsson, R. Wallny, D.H. Zhu

\section{Universität Zürich, Zurich, Switzerland}

T.K. Aarrestad, C. Amsler ${ }^{50}$, D. Brzhechko, M.F. Canelli, A. De Cosa, R. Del Burgo, B. Kilminster, S. Leontsinis, V.M. Mikuni, I. Neutelings, G. Rauco, P. Robmann, K. Schweiger, C. Seitz, Y. Takahashi, S. Wertz, A. Zucchetta

National Central University, Chung-Li, Taiwan

T.H. Doan, C.M. Kuo, W. Lin, A. Roy, S.S. Yu

\section{National Taiwan University (NTU), Taipei, Taiwan}

P. Chang, Y. Chao, K.F. Chen, P.H. Chen, W.-S. Hou, Y.y. Li, R.-S. Lu, E. Paganis, A. Psallidas, A. Steen

Chulalongkorn University, Faculty of Science, Department of Physics, Bangkok, Thailand

B. Asavapibhop, C. Asawatangtrakuldee, N. Srimanobhas, N. Suwonjandee 
Çukurova University, Physics Department, Science and Art Faculty, Adana, Turkey

A. Bat, F. Boran, A. Celik ${ }^{51}$, S. Cerci ${ }^{52}$, S. Damarseckin ${ }^{53}$, Z.S. Demiroglu, F. Dolek, C. Dozen ${ }^{54}$, I. Dumanoglu, G. Gokbulut, EmineGurpinar Guler ${ }^{55}$, Y. Guler, I. Hos ${ }^{56}$, C. Isik, E.E. Kangal ${ }^{57}$, O. Kara, A. Kayis Topaksu, U. Kiminsu, G. Onengut, K. Ozdemir ${ }^{58}$, S. Ozturk ${ }^{59}$, A.E. Simsek, D. Sunar Cerci ${ }^{52}$, U.G. Tok, S. Turkcapar, I.S. Zorbakir, C. Zorbilmez

Middle East Technical University, Physics Department, Ankara, Turkey B. Isildak ${ }^{60}$, G. Karapinar ${ }^{61}$, M. Yalvac

Bogazici University, Istanbul, Turkey

I.O. Atakisi, E. Gülmez, M. Kaya ${ }^{62}$, O. Kaya ${ }^{63}$, Ö. Özçelik, S. Tekten, E.A. Yetkin ${ }^{64}$

Istanbul Technical University, Istanbul, Turkey

A. Cakir, K. Cankocak, Y. Komurcu, S. Sen ${ }^{65}$

Istanbul University, Istanbul, Turkey

B. Kaynak, S. Ozkorucuklu

Institute for Scintillation Materials of National Academy of Science of Ukraine, Kharkov, Ukraine

B. Grynyov

National Scientific Center, Kharkov Institute of Physics and Technology, Kharkov, Ukraine

L. Levchuk

University of Bristol, Bristol, United Kingdom

E. Bhal, S. Bologna, J.J. Brooke, D. Burns ${ }^{66}$, E. Clement, D. Cussans, H. Flacher, J. Goldstein, G.P. Heath, H.F. Heath, L. Kreczko, B. Krikler, S. Paramesvaran, B. Penning, T. Sakuma, S. Seif El Nasr-Storey, V.J. Smith, J. Taylor, A. Titterton

Rutherford Appleton Laboratory, Didcot, United Kingdom

K.W. Bell, A. Belyaev ${ }^{67}$, C. Brew, R.M. Brown, D.J.A. Cockerill, J.A. Coughlan, K. Harder, S. Harper, J. Linacre, K. Manolopoulos, D.M. Newbold, E. Olaiya, D. Petyt, T. Reis, T. Schuh, C.H. Shepherd-Themistocleous, A. Thea, I.R. Tomalin, T. Williams, W.J. Womersley

\section{Imperial College, London, United Kingdom}

R. Bainbridge, P. Bloch, J. Borg, S. Breeze, O. Buchmuller, A. Bundock, GurpreetSingh CHAHAL ${ }^{68}$, D. Colling, P. Dauncey, G. Davies, M. Della Negra, R. Di Maria, P. Everaerts, G. Hall, G. Iles, M. Komm, C. Laner, L. Lyons, A.-M. Magnan, S. Malik, A. Martelli, V. Milosevic, A. Morton, J. Nash ${ }^{69}$, V. Palladino, M. Pesaresi, D.M. Raymond, A. Richards, A. Rose, E. Scott, C. Seez, A. Shtipliyski, M. Stoye, T. Strebler, A. Tapper, K. Uchida, T. Virdee ${ }^{16}$, N. Wardle, D. Winterbottom, J. Wright, A.G. Zecchinelli, S.C. Zenz 
Brunel University, Uxbridge, United Kingdom

J.E. Cole, P.R. Hobson, A. Khan, P. Kyberd, C.K. Mackay, I.D. Reid, L. Teodorescu, S. Zahid

Baylor University, Waco, U.S.A.

K. Call, B. Caraway, J. Dittmann, K. Hatakeyama, C. Madrid, B. McMaster, N. Pastika, C. Smith

Catholic University of America, Washington, DC, U.S.A.

R. Bartek, A. Dominguez, R. Uniyal, A.M. Vargas Hernandez

The University of Alabama, Tuscaloosa, U.S.A.

A. Buccilli, S.I. Cooper, C. Henderson, P. Rumerio, C. West

Boston University, Boston, U.S.A.

A. Albert, D. Arcaro, Z. Demiragli, D. Gastler, C. Richardson, J. Rohlf, D. Sperka, I. Suarez, L. Sulak, D. Zou

Brown University, Providence, U.S.A.

G. Benelli, B. Burkle, X. Coubez ${ }^{17}$, D. Cutts, Y.t. Duh, M. Hadley, U. Heintz, J.M. $\operatorname{Hogan}^{70}$, K.H.M. Kwok, E. Laird, G. Landsberg, K.T. Lau, J. Lee, Z. Mao, M. Narain, S. Sagir ${ }^{71}$, R. Syarif, E. Usai, D. Yu, W. Zhang

University of California, Davis, Davis, U.S.A.

R. Band, C. Brainerd, R. Breedon, M. Calderon De La Barca Sanchez, M. Chertok, J. Conway, R. Conway, P.T. Cox, R. Erbacher, C. Flores, G. Funk, F. Jensen, W. Ko, O. Kukral, R. Lander, M. Mulhearn, D. Pellett, J. Pilot, M. Shi, D. Taylor, K. Tos, M. Tripathi, Z. Wang, F. Zhang

University of California, Los Angeles, U.S.A.

M. Bachtis, C. Bravo, R. Cousins, A. Dasgupta, A. Florent, J. Hauser, M. Ignatenko, N. Mccoll, W.A. Nash, S. Regnard, D. Saltzberg, C. Schnaible, B. Stone, V. Valuev

University of California, Riverside, Riverside, U.S.A.

K. Burt, Y. Chen, R. Clare, J.W. Gary, S.M.A. Ghiasi Shirazi, G. Hanson, G. Karapostoli, E. Kennedy, O.R. Long, M. Olmedo Negrete, M.I. Paneva, W. Si, L. Wang, S. Wimpenny, B.R. Yates, Y. Zhang

University of California, San Diego, La Jolla, U.S.A.

J.G. Branson, P. Chang, S. Cittolin, S. Cooperstein, N. Deelen, M. Derdzinski, R. Gerosa, D. Gilbert, B. Hashemi, D. Klein, V. Krutelyov, J. Letts, M. Masciovecchio, S. May, S. Padhi, M. Pieri, V. Sharma, M. Tadel, F. Würthwein, A. Yagil, G. Zevi Della Porta

University of California, Santa Barbara - Department of Physics, Santa Barbara, U.S.A.

N. Amin, R. Bhandari, C. Campagnari, M. Citron, V. Dutta, M. Franco Sevilla, J. Incandela, B. Marsh, H. Mei, A. Ovcharova, H. Qu, J. Richman, U. Sarica, D. Stuart, S. Wang 
California Institute of Technology, Pasadena, U.S.A.

D. Anderson, A. Bornheim, O. Cerri, I. Dutta, J.M. Lawhorn, N. Lu, J. Mao, H.B. Newman, T.Q. Nguyen, J. Pata, M. Spiropulu, J.R. Vlimant, S. Xie, Z. Zhang, R.Y. Zhu

Carnegie Mellon University, Pittsburgh, U.S.A.

M.B. Andrews, T. Ferguson, T. Mudholkar, M. Paulini, M. Sun, I. Vorobiev, M. Weinberg University of Colorado Boulder, Boulder, U.S.A.

J.P. Cumalat, W.T. Ford, E. MacDonald, T. Mulholland, R. Patel, A. Perloff, K. Stenson, K.A. Ulmer, S.R. Wagner

Cornell University, Ithaca, U.S.A.

J. Alexander, Y. Cheng, J. Chu, A. Datta, A. Frankenthal, K. Mcdermott, J.R. Patterson, D. Quach, A. Ryd, S.M. Tan, Z. Tao, J. Thom, P. Wittich, M. Zientek

Fermi National Accelerator Laboratory, Batavia, U.S.A.

S. Abdullin, M. Albrow, M. Alyari, G. Apollinari, A. Apresyan, A. Apyan, S. Banerjee, L.A.T. Bauerdick, A. Beretvas, D. Berry, J. Berryhill, P.C. Bhat, K. Burkett, J.N. Butler, A. Canepa, G.B. Cerati, H.W.K. Cheung, F. Chlebana, M. Cremonesi, J. Duarte, V.D. Elvira, J. Freeman, Z. Gecse, E. Gottschalk, L. Gray, D. Green, S. Grünendahl, O. Gutsche, AllisonReinsvold Hall, J. Hanlon, R.M. Harris, S. Hasegawa, R. Heller, J. Hirschauer, B. Jayatilaka, S. Jindariani, M. Johnson, U. Joshi, T. Klijnsma, B. Klima, M.J. Kortelainen, B. Kreis, S. Lammel, J. Lewis, D. Lincoln, R. Lipton, M. Liu, T. Liu, J. Lykken, K. Maeshima, J.M. Marraffino, D. Mason, P. McBride, P. Merkel, S. Mrenna, S. Nahn, V. O'Dell, V. Papadimitriou, K. Pedro, C. Pena, G. Rakness, F. Ravera, L. Ristori, B. Schneider, E. Sexton-Kennedy, N. Smith, A. Soha, W.J. Spalding, L. Spiegel, S. Stoynev, J. Strait, N. Strobbe, L. Taylor, S. Tkaczyk, N.V. Tran, L. Uplegger, E.W. Vaandering, C. Vernieri, R. Vidal, M. Wang, H.A. Weber

University of Florida, Gainesville, U.S.A.

D. Acosta, P. Avery, D. Bourilkov, A. Brinkerhoff, L. Cadamuro, A. Carnes, V. Cherepanov, F. Errico, R.D. Field, S.V. Gleyzer, B.M. Joshi, M. Kim, J. Konigsberg, A. Korytov, K.H. Lo, P. Ma, K. Matchev, N. Menendez, G. Mitselmakher, D. Rosenzweig, K. Shi, J. Wang, S. Wang, X. Zuo

Florida International University, Miami, U.S.A.

Y.R. Joshi

Florida State University, Tallahassee, U.S.A.

T. Adams, A. Askew, S. Hagopian, V. Hagopian, K.F. Johnson, R. Khurana, T. Kolberg, G. Martinez, T. Perry, H. Prosper, C. Schiber, R. Yohay, J. Zhang

Florida Institute of Technology, Melbourne, U.S.A.

M.M. Baarmand, M. Hohlmann, D. Noonan, M. Rahmani, M. Saunders, F. Yumiceva

University of Illinois at Chicago (UIC), Chicago, U.S.A.

M.R. Adams, L. Apanasevich, R.R. Betts, R. Cavanaugh, X. Chen, S. Dittmer, O. Evdokimov, C.E. Gerber, D.A. Hangal, D.J. Hofman, K. Jung, C. Mills, T. Roy, M.B. Tonjes, N. Varelas, J. Viinikainen, H. Wang, X. Wang, Z. Wu 
The University of Iowa, Iowa City, U.S.A.

M. Alhusseini, B. Bilki ${ }^{55}$, W. Clarida, K. Dilsiz ${ }^{72}$, S. Durgut, R.P. Gandrajula, M. Haytmyradov, V. Khristenko, O.K. Köseyan, J.-P. Merlo, A. Mestvirishvili ${ }^{73}$, A. Moeller, J. Nachtman, H. Ogul ${ }^{74}$, Y. Onel, F. Ozok ${ }^{75}$, A. Penzo, C. Snyder, E. Tiras, J. Wetzel

Johns Hopkins University, Baltimore, U.S.A.

B. Blumenfeld, A. Cocoros, N. Eminizer, A.V. Gritsan, W.T. Hung, S. Kyriacou, P. Maksimovic, J. Roskes, M. Swartz

The University of Kansas, Lawrence, U.S.A.

C. Baldenegro Barrera, P. Baringer, A. Bean, S. Boren, J. Bowen, A. Bylinkin,

T. Isidori, S. Khalil, J. King, G. Krintiras, A. Kropivnitskaya, C. Lindsey, D. Majumder,

W. Mcbrayer, N. Minafra, M. Murray, C. Rogan, C. Royon, S. Sanders, E. Schmitz, J.D. Tapia Takaki, Q. Wang, J. Williams, G. Wilson

Kansas State University, Manhattan, U.S.A.

S. Duric, A. Ivanov, K. Kaadze, D. Kim, Y. Maravin, D.R. Mendis, T. Mitchell, A. Modak, A. Mohammadi

Lawrence Livermore National Laboratory, Livermore, U.S.A.

F. Rebassoo, D. Wright

University of Maryland, College Park, U.S.A.

A. Baden, O. Baron, A. Belloni, S.C. Eno, Y. Feng, N.J. Hadley, S. Jabeen, G.Y. Jeng, R.G. Kellogg, J. Kunkle, A.C. Mignerey, S. Nabili, F. Ricci-Tam, M. Seidel, Y.H. Shin, A. Skuja, S.C. Tonwar, K. Wong

Massachusetts Institute of Technology, Cambridge, U.S.A.

D. Abercrombie, B. Allen, A. Baty, R. Bi, S. Brandt, W. Busza, I.A. Cali, M. D'Alfonso,

G. Gomez Ceballos, M. Goncharov, P. Harris, D. Hsu, M. Hu, M. Klute, D. Kovalskyi, Y.-J. Lee, P.D. Luckey, B. Maier, A.C. Marini, C. Mcginn, C. Mironov, S. Narayanan, X. Niu, C. Paus, D. Rankin, C. Roland, G. Roland, Z. Shi, G.S.F. Stephans, K. Sumorok, K. Tatar, D. Velicanu, J. Wang, T.W. Wang, B. Wyslouch

University of Minnesota, Minneapolis, U.S.A.

R.M. Chatterjee, A. Evans, S. Guts ${ }^{\dagger}$, P. Hansen, J. Hiltbrand, Sh. Jain, Y. Kubota, Z. Lesko, J. Mans, M. Revering, R. Rusack, R. Saradhy, N. Schroeder, M.A. Wadud

University of Mississippi, Oxford, U.S.A.

J.G. Acosta, S. Oliveros

University of Nebraska-Lincoln, Lincoln, U.S.A.

K. Bloom, S. Chauhan, D.R. Claes, C. Fangmeier, L. Finco, F. Golf, R. Kamalieddin, I. Kravchenko, J.E. Siado, G.R. Snow ${ }^{\dagger}$, B. Stieger, W. Tabb

State University of New York at Buffalo, Buffalo, U.S.A.

G. Agarwal, C. Harrington, I. Iashvili, A. Kharchilava, C. McLean, D. Nguyen, A. Parker, J. Pekkanen, S. Rappoccio, B. Roozbahani 
Northeastern University, Boston, U.S.A.

G. Alverson, E. Barberis, C. Freer, Y. Haddad, A. Hortiangtham, G. Madigan, B. Marzocchi, D.M. Morse, T. Orimoto, L. Skinnari, A. Tishelman-Charny, T. Wamorkar, B. Wang, A. Wisecarver, D. Wood

Northwestern University, Evanston, U.S.A.

S. Bhattacharya, J. Bueghly, T. Gunter, K.A. Hahn, N. Odell, M.H. Schmitt, K. Sung, M. Trovato, M. Velasco

University of Notre Dame, Notre Dame, U.S.A.

R. Bucci, N. Dev, R. Goldouzian, M. Hildreth, K. Hurtado Anampa, C. Jessop, D.J. Karmgard, K. Lannon, W. Li, N. Loukas, N. Marinelli, I. Mcalister, F. Meng, C. Mueller, Y. Musienko ${ }^{37}$, M. Planer, R. Ruchti, P. Siddireddy, G. Smith, S. Taroni, M. Wayne, A. Wightman, M. Wolf, A. Woodard

The Ohio State University, Columbus, U.S.A.

J. Alimena, B. Bylsma, L.S. Durkin, B. Francis, C. Hill, W. Ji, A. Lefeld, T.Y. Ling, B.L. Winer

Princeton University, Princeton, U.S.A.

G. Dezoort, P. Elmer, J. Hardenbrook, N. Haubrich, S. Higginbotham, A. Kalogeropoulos, S. Kwan, D. Lange, M.T. Lucchini, J. Luo, D. Marlow, K. Mei, I. Ojalvo, J. Olsen, C. Palmer, P. Piroué, J. Salfeld-Nebgen, D. Stickland, C. Tully, Z. Wang

University of Puerto Rico, Mayaguez, U.S.A.

S. Malik, S. Norberg

Purdue University, West Lafayette, U.S.A.

A. Barker, V.E. Barnes, S. Das, L. Gutay, M. Jones, A.W. Jung, A. Khatiwada, B. Mahakud, D.H. Miller, G. Negro, N. Neumeister, C.C. Peng, S. Piperov, H. Qiu, J.F. Schulte, N. Trevisani, F. Wang, R. Xiao, W. Xie

Purdue University Northwest, Hammond, U.S.A.

T. Cheng, J. Dolen, N. Parashar

Rice University, Houston, U.S.A.

U. Behrens, K.M. Ecklund, S. Freed, F.J.M. Geurts, M. Kilpatrick, Arun Kumar, W. Li, B.P. Padley, R. Redjimi, J. Roberts, J. Rorie, W. Shi, A.G. Stahl Leiton, Z. Tu, A. Zhang

University of Rochester, Rochester, U.S.A.

A. Bodek, P. de Barbaro, R. Demina, J.L. Dulemba, C. Fallon, T. Ferbel, M. Galanti, A. Garcia-Bellido, O. Hindrichs, A. Khukhunaishvili, E. Ranken, R. Taus

Rutgers, The State University of New Jersey, Piscataway, U.S.A.

B. Chiarito, J.P. Chou, A. Gandrakota, Y. Gershtein, E. Halkiadakis, A. Hart, M. Heindl,

E. Hughes, S. Kaplan, I. Laflotte, A. Lath, R. Montalvo, K. Nash, M. Osherson, H. Saka,

S. Salur, S. Schnetzer, S. Somalwar, R. Stone, S. Thomas 
University of Tennessee, Knoxville, U.S.A.

H. Acharya, A.G. Delannoy, S. Spanier

Texas A\&M University, College Station, U.S.A.

O. Bouhali ${ }^{76}$, M. Dalchenko, M. De Mattia, A. Delgado, S. Dildick, R. Eusebi, J. Gilmore, T. Huang, T. Kamon ${ }^{77}$, S. Luo, S. Malhotra, D. Marley, R. Mueller, D. Overton, L. Perniè,

D. Rathjens, A. Safonov

Texas Tech University, Lubbock, U.S.A.

N. Akchurin, J. Damgov, F. De Guio, S. Kunori, K. Lamichhane, S.W. Lee, T. Mengke, S. Muthumuni, T. Peltola, S. Undleeb, I. Volobouev, Z. Wang, A. Whitbeck

Vanderbilt University, Nashville, U.S.A.

S. Greene, A. Gurrola, R. Janjam, W. Johns, C. Maguire, A. Melo, H. Ni, K. Padeken, F. Romeo, P. Sheldon, S. Tuo, J. Velkovska, M. Verweij

University of Virginia, Charlottesville, U.S.A.

M.W. Arenton, P. Barria, B. Cox, G. Cummings, J. Hakala, R. Hirosky, M. Joyce, A. Ledovskoy, C. Neu, B. Tannenwald, Y. Wang, E. Wolfe, F. Xia

Wayne State University, Detroit, U.S.A.

R. Harr, P.E. Karchin, N. Poudyal, J. Sturdy, P. Thapa

University of Wisconsin - Madison, Madison, WI, U.S.A.

T. Bose, J. Buchanan, C. Caillol, D. Carlsmith, S. Dasu, I. De Bruyn, L. Dodd, F. Fiori, C. Galloni, H. He, M. Herndon, A. Hervé, U. Hussain, P. Klabbers, A. Lanaro, A. Loeliger, K. Long, R. Loveless, J. Madhusudanan Sreekala, D. Pinna, T. Ruggles, A. Savin, V. Sharma, W.H. Smith, D. Teague, S. Trembath-reichert, N. Woods

$\dagger$ : Deceased

1: Also at Vienna University of Technology, Vienna, Austria

2: Also at IRFU, CEA, Université Paris-Saclay, Gif-sur-Yvette, France

3: Also at Universidade Estadual de Campinas, Campinas, Brazil

4: Also at Federal University of Rio Grande do Sul, Porto Alegre, Brazil

5: Also at UFMS, Nova Andradina, Brazil

6: Also at Universidade Federal de Pelotas, Pelotas, Brazil

7: Also at Université Libre de Bruxelles, Bruxelles, Belgium

8: Also at University of Chinese Academy of Sciences, Beijing, China

9: Also at Institute for Theoretical and Experimental Physics named by A.I. Alikhanov of NRC 'Kurchatov Institute', Moscow, Russia

10: Also at Joint Institute for Nuclear Research, Dubna, Russia

11: Also at Cairo University, Cairo, Egypt

12: Now at British University in Egypt, Cairo, Egypt

13: Also at Purdue University, West Lafayette, U.S.A.

14: Also at Université de Haute Alsace, Mulhouse, France

15: Also at Erzincan Binali Yildirim University, Erzincan, Turkey

16: Also at CERN, European Organization for Nuclear Research, Geneva, Switzerland

17: Also at RWTH Aachen University, III. Physikalisches Institut A, Aachen, Germany

18: Also at University of Hamburg, Hamburg, Germany 
19: Also at Brandenburg University of Technology, Cottbus, Germany

20: Also at Institute of Physics, University of Debrecen, Debrecen, Hungary, Debrecen, Hungary

21: Also at Institute of Nuclear Research ATOMKI, Debrecen, Hungary

22: Also at MTA-ELTE Lendület CMS Particle and Nuclear Physics Group, Eötvös Loránd University, Budapest, Hungary, Budapest, Hungary

23: Also at IIT Bhubaneswar, Bhubaneswar, India, Bhubaneswar, India

24: Also at Institute of Physics, Bhubaneswar, India

25: Also at Shoolini University, Solan, India

26: Also at University of Hyderabad, Hyderabad, India

27: Also at University of Visva-Bharati, Santiniketan, India

28: Also at Isfahan University of Technology, Isfahan, Iran

29: Now at INFN Sezione di Bari ${ }^{a}$, Università di Bari ${ }^{b}$, Politecnico di Bari ${ }^{c}$, Bari, Italy

30: Also at Italian National Agency for New Technologies, Energy and Sustainable Economic Development, Bologna, Italy

31: Also at Centro Siciliano di Fisica Nucleare e di Struttura Della Materia, Catania, Italy

32: Also at Scuola Normale e Sezione dell'INFN, Pisa, Italy

33: Also at Riga Technical University, Riga, Latvia, Riga, Latvia

34: Also at Malaysian Nuclear Agency, MOSTI, Kajang, Malaysia

35: Also at Consejo Nacional de Ciencia y Tecnología, Mexico City, Mexico

36: Also at Warsaw University of Technology, Institute of Electronic Systems, Warsaw, Poland

37: Also at Institute for Nuclear Research, Moscow, Russia

38: Now at National Research Nuclear University 'Moscow Engineering Physics Institute' (MEPhI), Moscow, Russia

39: Also at St. Petersburg State Polytechnical University, St. Petersburg, Russia

40: Also at University of Florida, Gainesville, U.S.A.

41: Also at Imperial College, London, United Kingdom

42: Also at P.N. Lebedev Physical Institute, Moscow, Russia

43: Also at California Institute of Technology, Pasadena, U.S.A.

44: Also at Budker Institute of Nuclear Physics, Novosibirsk, Russia

45: Also at Faculty of Physics, University of Belgrade, Belgrade, Serbia

46: Also at Università degli Studi di Siena, Siena, Italy

47: Also at INFN Sezione di Pavia ${ }^{a}$, Università di Pavia ${ }^{b}$, Pavia, Italy, Pavia, Italy

48: Also at National and Kapodistrian University of Athens, Athens, Greece

49: Also at Universität Zürich, Zurich, Switzerland

50: Also at Stefan Meyer Institute for Subatomic Physics, Vienna, Austria, Vienna, Austria

51: Also at Burdur Mehmet Akif Ersoy University, BURDUR, Turkey

52: Also at Adiyaman University, Adiyaman, Turkey

53: Also at Şırnak University, Sirnak, Turkey

54: Also at Department of Physics, Tsinghua University, Beijing, China, Beijing, China

55: Also at Beykent University, Istanbul, Turkey, Istanbul, Turkey

56: Also at Istanbul Aydin University, Application and Research Center for Advanced Studies (App. \& Res. Cent. for Advanced Studies), Istanbul, Turkey

57: Also at Mersin University, Mersin, Turkey

58: Also at Piri Reis University, Istanbul, Turkey

59: Also at Gaziosmanpasa University, Tokat, Turkey

60: Also at Ozyegin University, Istanbul, Turkey

61: Also at Izmir Institute of Technology, Izmir, Turkey

62: Also at Marmara University, Istanbul, Turkey 
63: Also at Kafkas University, Kars, Turkey

64: Also at Istanbul Bilgi University, Istanbul, Turkey

65: Also at Hacettepe University, Ankara, Turkey

66: Also at Vrije Universiteit Brussel, Brussel, Belgium

67: Also at School of Physics and Astronomy, University of Southampton, Southampton, United Kingdom

68: Also at IPPP Durham University, Durham, United Kingdom

69: Also at Monash University, Faculty of Science, Clayton, Australia

70: Also at Bethel University, St. Paul, Minneapolis, U.S.A., St. Paul, U.S.A.

71: Also at Karamanoğlu Mehmetbey University, Karaman, Turkey

72: Also at Bingol University, Bingol, Turkey

73: Also at Georgian Technical University, Tbilisi, Georgia

74: Also at Sinop University, Sinop, Turkey

75: Also at Mimar Sinan University, Istanbul, Istanbul, Turkey

76: Also at Texas A\&M University at Qatar, Doha, Qatar

77: Also at Kyungpook National University, Daegu, Korea, Daegu, Korea 